\title{
Potential functions on Grassmannians of planes and cluster transformations
}

\author{
Yuichi Nohara, Kazushi Ueda
}

\begin{abstract}
With a triangulation of a planar polygon with $n$ sides, one can associate an integrable system on the Grassmannian of 2-planes in an $n$-space. In this paper, we show that the potential functions of Lagrangian torus fibers of the integrable systems associated with different triangulations glue together by cluster transformations. We also prove that the cluster transformations coincide with the wall-crossing formula in Lagrangian intersection Floer theory.
\end{abstract}

\section{Introduction}

Quantum cohomologies of Grassmannians give quantum deformations of the classical Schubert calculus. It is a fascinating subject, which is related to many branch of mathematics such as moduli of vector bundles on a Riemann surface [Wit95], total positivity [Rie01], and eigenvalue problems [TW03] to name a few.

Mirror symmetry is a powerful tool to study quantum cohomologies of symplectic manifolds. The mirror of a Fano manifold is a Landau-Ginzburg model, i.e., a pair $(\check{X}, W)$ of an analytic space $\check{X}$ and an analytic function $W: \check{X} \rightarrow \mathbb{A}^{1}$ called the Landau-Ginzburg potential. Landau-Ginzburg mirrors of flag varieties are introduced in [Rie08], where $\check{X}$ are the complements of anti-canonical divisors in the flag manifolds associated with the Langlands dual groups, and $W$ are regular functions. In the type $A$ cases, the restrictions of $W$ to certain open subvarieties give mirrors introduced earlier in [EHX97, BCFKvS00]. For the Grassmannian $\operatorname{Gr}(k, n)=\operatorname{Gr}\left(k, \mathbb{C}^{n}\right)$ of $k$ dimensional subspaces in $\mathbb{C}^{n}$, Marsh and Rietsch [MR give a description of the Landau-Ginzburg mirror

$$
W: \check{X}=\operatorname{Gr}\left(n-k,\left(\mathbb{C}^{n}\right)^{*}\right) \backslash D \longrightarrow \mathbb{A}^{1}
$$

in terms of Plücker coordinates on the dual Grassmannian $\operatorname{Gr}\left(n-k,\left(\mathbb{C}^{n}\right)^{*}\right)$ of $\operatorname{Gr}\left(k, \mathbb{C}^{n}\right)$.

With a Lagrangian submanifold of a symplectic manifold, one can associate the potential function, which is a Floer-theoretic quantity obtained as 
the generating function of numbers of pseudo-holomorphic disks bounded by Lagrangian submanifolds [FOOO09]. In the case of toric manifolds, the potential functions of Lagrangian orbits of the torus action can be identified with the Landau-Ginzburg potentials of the mirrors.

In contrast to the toric cases where the toric moment maps give canonical Lagrangian torus fibrations, there are a priori no preferred Lagrangian torus fibrations on flag manifolds. In the case of the Grassmannian $\operatorname{Gr}(2, n)$ of 2 -planes, with any triangulation $\Gamma$ of a convex polygon with $n$ sides, one can associate a completely integrable system

$$
\Psi_{\Gamma}: \operatorname{Gr}(2, n) \longrightarrow \mathbb{R}^{2 n-4}
$$

whose image $\Delta_{\Gamma}=\Psi_{\Gamma}(\operatorname{Gr}(2, n))$ is a convex polytope. Note that the number of ways to triangulate a convex $n$-gon is given by the Catalan number $C_{n-2}=\frac{1}{n-1}\left(\begin{array}{c}2 n-4 \\ n-2\end{array}\right)$. The potential function of Lagrangian torus fibers of the integrable system $\Psi_{\Gamma}$ is computed in [NU14, Theorem 1.6]. It is written as a Laurent polynomial $W_{\Gamma}$, which gives a regular function on a torus $\left(\mathbb{G}_{m}\right)^{2 n-4}$.

If two triangulations $\Gamma$ and $\Gamma^{\prime}$ are related by a Whitehead move (see Figure 3.2, the corresponding potential functions $W_{\Gamma}$ and $W_{\Gamma^{\prime}}$ are related by a subtraction-free birational change of variables of the form

$$
W_{\Gamma^{\prime}}\left(\ldots, y^{\prime}, y_{1}, y_{2}, y_{3}, y_{4}, \ldots\right)=W_{\Gamma}\left(\ldots, y, y_{1}, y_{2}, y_{3}, y_{4}, \ldots\right)
$$

where

$$
y^{\prime}=\frac{1}{y} \cdot \frac{y_{1} y_{2} y_{3} y_{4}}{y_{1} y_{3}+y_{2} y_{4}} .
$$

Moreover, the tropicalization of this coordinate change gives a piecewiselinear transformation on $\mathbb{R}^{2 n-4}$ which maps $\Delta_{\Gamma}$ into $\Delta_{\Gamma^{\prime}}$.

In what follows we identify the dual Grassmannian $\operatorname{Gr}\left(n-2,\left(\mathbb{C}^{n}\right)^{*}\right)$ with $\operatorname{Gr}(2, n)$ in a canonical way, and write the Plücker coordinates as $p_{i j}$ $(1 \leq i<j \leq n)$. The first main result in this paper is the following:

Theorem 1.1. For any triangulation $\Gamma$, there is an open embedding

$$
\iota_{\Gamma}:\left(\mathbb{G}_{m}\right)^{2 n-4} \hookrightarrow \check{X}
$$

such that the restriction of the Landau-Ginzburg potential coincides with the potential function; $\iota_{\Gamma}^{*} W=W_{\Gamma}$. The change of variables (1.4) can be identified with the Plücker relation

$$
p_{i k} p_{j l}=p_{i j} p_{k l}+p_{i l} p_{j k}
$$

by a suitable choice of a coordinate on $\left(\mathbb{G}_{m}\right)^{2 n-4}$.

In other words, the potential functions for different triangulations glue together to form an open dense subset of Marsh-Rietsch's mirror. The Plücker relation (1.6) is a prototypical example of a cluster transformation in the theory of cluster algebras [FZ02]. 
Remark 1.2. Rietsch and Williams $[\mathrm{RW}]$ also study the relation between piecewise-linear transformations for "moment polytopes" $\Delta_{\Gamma}$ and cluster transformations (1.6) from a slightly different view point, where $\Delta_{\Gamma}$ are regarded as Newton-Okounkov bodies.

For a pair $\Gamma$ and $\Gamma^{\prime}$ of triangulations related by a Whitehead move, one can construct a one-parameter family $\Psi_{t}(0 \leq t \leq 1)$ of completely integrable systems on $\operatorname{Gr}(2, n)$ such that $\Psi_{0}=\Psi_{\Gamma}$ and $\Psi_{1}=\Psi_{\Gamma^{\prime}}$ (up to coordinate changes on the base spaces). For $t \neq 0,1$, the integrable system $\Psi_{t}$ has singular fibers over a codimension two subset in the interior of the base space $B_{t}=\Psi_{t}(\operatorname{Gr}(2, n))$. The presence of singular fibers leads to a codimension one wall in $B_{t}$ which divides $B_{t}$ into two chambers. The $S Y Z$ mirror in the sense of [AAK16, Definition 1.2] of $\operatorname{Gr}(2, n)$ with respect to this Lagrangian torus fibration is given by gluing (open subsets of) Landau-Ginzburg models $\left(\left(\mathbb{G}_{m}\right)^{2 n-4}, W_{\Gamma}\right)$ and $\left(\left(\mathbb{G}_{m}\right)^{2 n-4}, W_{\Gamma^{\prime}}\right)$ (and then completing). Each of these Landau-Ginzburg models comes from the moduli space of objects of the Fukaya category supported by Lagrangian torus fibers above each chamber, and the gluing is given by a wall-crossing formula obtained by counting pseudo-holomorphic disks of Maslov index zero. The second main result in this paper is the following:

Theorem 1.3. For $0<t<1$, the wall-crossing formula in the construction of the $S Y Z$ mirror of $\operatorname{Gr}(2, n)$ with respect to the Lagrangian torus fibration $\Psi_{t}$ is given by the coordinate change (1.4).

Theorem 1.3 is proved by reduction to the case of $\operatorname{Gr}(2,4)$ by a degeneration argument, which is then handled directly along the lines of Aur07, Aur09.

It is suggested in [Aur07, Aur09] that the mirror of a Fano manifold is obtained by first taking a special Lagrangian torus fibration on the complement of an anti-canonical divisor, and then equipping its Strominger-Yau-Zaslow mirror with the potential function of the fiber. The integrable system $\Psi_{t}$ does not restrict to a Lagrangian torus fibration on the complement of an anti-canonical divisor, and it is an interesting problem to find a Lagrangian torus fibration on the complement of an anti-canonical divisor, which allows one to fit Rietsch's mirror into this framework. Another interesting question is whether there are other mirrors associated with other Lagrangian torus fibrations on the complement of other anti-canonical divisors.

This paper is organized as follows. After fixing notation for triangulations of convex polygons in Section 2, we give in Section 3 the construction of completely integrable systems on $\operatorname{Gr}(2, n)$. In Section 4 we recall toric degenerations of $\operatorname{Gr}(2, n)$ associated with triangulations of a convex $n$-gon, which enables us to compute potential functions of Lagrangian torus fibers of $\Psi_{\Gamma}$. In Section 5 we show that the potential functions for different triangulations are related by the coordinate change (1.4). Section 6 is a quick 
review of cluster algebras. Theorem 1.1 is proved in Section 7. In Section 8 we recall the wall-crossing formula given by Auroux in Aur07, Aur09, which is enough for our purpose since the integrable system $\Psi_{t}$ has only one wall. In Section 9 we prove Theorem 1.3 in the case of $\operatorname{Gr}(2,4)$. The proof for general $\operatorname{Gr}(2, n)$ is given in Section 10 .

Acknowledgment: We thank Yankı Lekili for collaboration at an early stage of this work; it is originally conceived as a joint project with him. We thank River Chiang for organizing a workshop in Tainan in July 2014, where this project has been initiated. We also thank the anonymous referee for reading the manuscript carefully, pointing out mistakes, and suggesting a number of improvements. Y. N. is supported by Grant-in-Aid for Scientific Research (15K04847). K. U. is supported by Grant-in-Aid for Scientific Research (24740043, 15KT0105, 16K13743, 16H03930).

\section{Triangulations}

Fix an integer $n$ greater than 2 , and let $P$ be a convex planar polygon with $n$ sides called the reference polygon. We order the vertices of $P$ in such a way that respects the natural cyclic order on the boundary of $P$, and define the side vectors $e_{i} \in \mathbb{R}^{2}$ for $i=1, \ldots, n$ as the difference between the $i$-th vertex and the $(i+1)$-st vertex. Setting $I(i, j)=\{i, i+1, i+2, \ldots, j-1\}$, we can write the diagonal connecting the $i$-th vertex and the $j$-th vertex as

$$
d_{i j}=\sum_{k \in I(i, j)} e_{k}
$$

Take a subdivision $\Gamma$ of $P$ given by a set of diagonals which are pairwise non-crossing in the interior of $P$. Note that the non-crossing condition for diagonals $d_{i j}, d_{k l}$ is equivalent to

$$
I(i, j) \subset I(k, l) \text { or } I(k, l) \subset I(i, j) \text { or } I(i, j) \cap I(k, l)=\emptyset .
$$

We consider the dual graph of the subdivision $\Gamma$, which is a tree with $n$ leaves. Let $\epsilon(i, j)$ denote an edge in the graph intersecting a diagonal $d_{i j}$ or a side $e_{i}=d_{i, i+1}$ of $P$ connecting the $i$-th and $j$-th vertices, where we assume $\epsilon(n, n+1)=\epsilon(1, n)$. In what follows we regard $\Gamma$ as the set of edges in the dual graph by abuse of notation, and let

$$
\begin{aligned}
\operatorname{Int} \Gamma & =\{\epsilon(i, j) \in \Gamma|| i-j \mid \geq 2\}, \\
\partial \Gamma & =\{\epsilon(i, i+1) \mid i=1, \ldots, n\}
\end{aligned}
$$

be the sets of interior edges and leaves of $\Gamma$, respectively. We also consider a "pruned" tree obtained from $\Gamma$ by removing the $n$-th leaf $\epsilon(1, n)$, and let

$$
\operatorname{Prn} \Gamma=\Gamma \backslash\{\epsilon(1, n)\}
$$




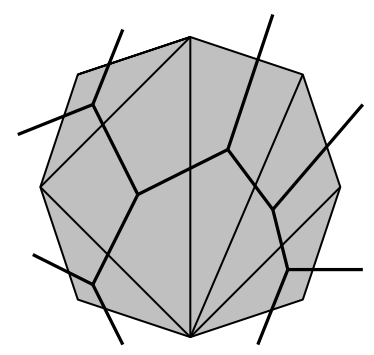

Figure 2.1: A triangulation of a convex polygon and its dual graph.

be the set of its edges. If $\Gamma$ is a triangulation of $P$, which is given by $n-3$ diagonals, then we have

$$
\begin{aligned}
\# \Gamma & =2 n-3, \\
\# \partial \Gamma & =n=\operatorname{dim}_{\mathbb{T}_{U(n)},} \\
\# \operatorname{Prn} \Gamma & =2 n-4=\operatorname{dim}_{\mathbb{C}} \operatorname{Gr}(2, n), \\
\# \operatorname{Int} \Gamma & =n-3=\operatorname{dim}_{\mathbb{C}} \operatorname{Gr}(2, n) / / \mathbb{T}_{U(n)},
\end{aligned}
$$

where $\mathbb{T}_{U(n)} \subset U(n)$ is a maximal torus consisting of diagonal matrices.

\section{Integrable systems on $\operatorname{Gr}(2, n)$}

Fix a constant $\lambda>0$, and identify the Grassmannian $\operatorname{Gr}(2, n)$ of 2-planes in $\mathbb{C}^{n}$ with the adjoint orbit

$$
\mathcal{O}_{\lambda}=\{x \in \sqrt{-1} \mathfrak{u}(n) \mid \text { eigenvalues of } x \text { are } \lambda, \lambda, 0, \ldots, 0\}
$$

of a diagonal matrix $\operatorname{diag}(\lambda, \lambda, 0, \ldots, 0)$ in the space $\sqrt{-1} \mathfrak{u}(n)$ of Hermitian matrices. For each $1 \leq i<j \leq n+1$, we consider the adjoint action on $\mathcal{O}_{\lambda}$ of the subgroup

$$
G(i, j)=\left(\begin{array}{ccc}
\mathbf{1}_{i-1} & & \\
& U(j-i) & \\
& & \mathbf{1}_{n-j+1}
\end{array}\right) \cong U(j-i)
$$

of $U(n)$. Its moment map is given by

$$
\mu_{G(i, j)}: \mathcal{O}_{\lambda} \rightarrow \sqrt{-1} \mathfrak{u}(j-i), \quad x=\left(x_{k l}\right) \mapsto \mu_{G(i, j)}(x)=\left(x_{k l}\right)_{k, l \in I(i, j)},
$$

where we identify the dual space of the Lie algebra $\operatorname{Lie} G(i, j) \cong \mathfrak{u}(j-i)$ of $G(i, j)$ with $\sqrt{-1} \mathfrak{u}(j-i)$ by an invariant inner product. Since

$$
\operatorname{rank} \mu_{G(i, j)}(x) \leq \operatorname{rank} x \leq 2, \quad x \in \mathcal{O}_{\lambda}
$$


for each $(i, j)$ with $|i-j| \geq 2$, each Hermitian matrix $\mu_{G(i, j)}(x)$ has at most two nonzero eigenvalues $\lambda_{1}^{(i, j)}(x) \geq \lambda_{2}^{(i, j)}(x) \geq 0$. For each $1 \leq i<j \leq n$, we define a function $\psi_{i j}$ on $\mathcal{O}_{\lambda}$ by

$$
\psi_{i j}(x)= \begin{cases}\lambda_{1}^{(i, j)}(x), & \text { if }|i-j| \geq 2, \\ \mu_{G(i, i+1)}(x)=x_{i i}, & j=i+1 .\end{cases}
$$

Note that the the moment map $\mu_{\mathbb{T}_{U(n)}}: \mathcal{O}_{\lambda} \rightarrow \mathbb{R}^{n}$ of the action of the maximal torus $\mathbb{T}_{U(n)}=\prod_{i=1}^{n} G(i, i+1)$ is given by

$$
\mu_{\mathbb{T}_{U(n)}}=\left(\psi_{12}, \psi_{23}, \psi_{34}, \ldots, \psi_{n, n+1}\right),
$$

and hence $\left\{\psi_{i, i+1}\right\}_{1 \leq i \leq n}$ satisfies one relation

$$
\psi_{12}(x)+\psi_{23}(x)+\cdots+\psi_{n-1, n}(x)+\psi_{n, n+1}(x)=\operatorname{tr} x=2 \lambda .
$$

In general, for any $n \times n$ Hermitian matrix $x=\left(x_{i j}\right)$, the mini-max principle implies that the eigenvalues $\lambda_{1} \geq \cdots \geq \lambda_{n}$ of $x$ and those $\mu_{1} \geq \cdots \geq \mu_{n-1}$ of the submatrix $\left(x_{i j}\right)_{1 \leq i, j \leq n-1}$ satisfy

$$
\lambda_{1} \geq \mu_{1} \geq \lambda_{2} \geq \mu_{2} \geq \lambda_{3} \geq \cdots \geq \lambda_{n-1} \geq \mu_{n-1} \geq \lambda_{n} .
$$

In our situation, each $x \in \mathcal{O}_{\lambda}$ has eigenvalues $\lambda, \lambda, 0, \ldots, 0$, and hence the largest eigenvalue $\psi_{1 n}(x)=\lambda_{1}^{(1, n)}(x)$ of $\mu_{G(1, n)}(x)=\left(x_{i j}\right)_{1 \leq i, j \leq n-1}$ is constant:

$$
\psi_{1 n}=\lambda .
$$

For a triangulation $\Gamma$ of the reference $n$-gon $P$, the non-crossing condition (2.2) implies that $G(i, j) \subset G(k, l)$ or $G(k, l) \subset G(i, j)$ or the actions of $G(i, j)$ and $G(k, l)$ on $\mathcal{O}_{\lambda}$ commute for each pair $\epsilon(i, j), \epsilon(k, l) \in \operatorname{Int} \Gamma$ of interior edges. By applying the construction of completely integrable systems in GS83, we obtain the following:

Theorem 3.1 ([NU14, Section 4]). For a triangulation $\Gamma$ of the reference polygon, the map

$$
\Psi_{\Gamma}=\left(\psi_{i j}\right)_{\epsilon(i, j) \in \operatorname{Prn} \Gamma}: \mathcal{O}_{\lambda} \rightarrow \mathbb{R}^{\operatorname{Prn} \Gamma}=\mathbb{R}^{2 n-4}
$$

is a completely integrable on $\operatorname{Gr}(2, n) \cong \mathcal{O}_{\lambda}$ with respect to the KostantKirillov form. The natural coordinate $\left(u_{i j}\right)_{\epsilon(i, j) \in \operatorname{Prn} \Gamma}$ on $\mathbb{R}^{\operatorname{Prn} \Gamma}$ gives an action coordinate, and the image $\Delta_{\Gamma}=\Psi_{\Gamma}\left(\mathcal{O}_{\lambda}\right)$ is a convex polytope.

Remark 3.2. One can apply this construction for general partial flag manifolds of type A to obtain several completely integrable systems. 
Remark 3.3. In [NU14], we defined $\psi_{i j}=\lambda_{2}^{(i, j)}$ for $|i-j| \geq 2$ instead of $\lambda_{1}^{(i, j)}$. Since

$$
\lambda_{1}^{(i, j)}+\lambda_{2}^{(i, j)}=\operatorname{tr} \mu_{G(i, j)}=\sum_{k=i}^{j-1} \psi_{k, k+1},
$$

the completely integrable system $\Psi_{\Gamma}$ in (3.10) and that in [NU14] are related by a linear transformation on the base space, and hence fibers of these integrable systems are the same.

To describe the polytope $\Delta_{\Gamma}$ explicitly, we recall bending Hamiltonians on polygon spaces introduced by Kapovich and Millson KM96 and Klyachko Kly94. For an $n$-tuple $\boldsymbol{r}=\left(r_{1}, \ldots, r_{n}\right) \in\left(\mathbb{R}^{>0}\right)^{n}$ of positive numbers, the polygon space $\mathcal{M}_{\boldsymbol{r}}$ is defined to be a moduli space of $n$-gons in $\mathbb{R}^{3}$ with fixed side lengths $r_{1}, \ldots, r_{n}$ :

$$
\mathcal{M}_{\boldsymbol{r}} \cong\left\{\boldsymbol{\xi}=\left(\xi_{1}, \ldots, \xi_{n}\right) \in \prod_{i=1}^{n} S^{2}\left(r_{i}\right) \mid \sum_{i=1}^{n} \xi_{i}=0\right\} / S O(3),
$$

where $S^{2}(r) \subset \mathbb{R}^{3}$ is a 2 -sphere of radius $r$ centered at the origin. For each $1 \leq i<j \leq n$, let $\varphi_{i j}: \mathcal{M}_{r} \rightarrow \mathbb{R}$ be the function which measures the length of the diagonal or the side of each polygon $\boldsymbol{\xi}$ connecting the $i$-th and $j$-th vertices:

$$
\varphi_{i j}(\boldsymbol{\xi})=\left|\xi_{i}+\xi_{i+1}+\cdots+\xi_{j-1}\right| .
$$

Note that $\varphi_{i, i+1}=r_{i}$ are constant functions. The function $\varphi_{i j}$ for $|i-j| \geq 2$ is called a bending Hamiltonian, since its Hamiltonian flow bends polygons $\boldsymbol{\xi} \in \mathcal{M}_{r}$ along the diagonal connecting the $i$-th and $j$-th vertices.

Theorem 3.4 (Kapovich and Millson [KM96], Klyachko [Kly94]). For each triangulation $\Gamma$ of $P$, the map

$$
\Phi_{\Gamma}=\left(\varphi_{i j}\right)_{\epsilon(i, j) \in \operatorname{Int} \Gamma}: \mathcal{M}_{\boldsymbol{r}} \rightarrow \mathbb{R}^{n-3}
$$

is a completely integrable system on $\mathcal{M}_{\boldsymbol{r}}$. The image $\Phi_{\Gamma}\left(\mathcal{M}_{\boldsymbol{r}}\right)$ is a convex polytope defined by triangle inequalities

$$
\left|\varphi_{i j}-\varphi_{j k}\right| \leq \varphi_{i k} \leq \varphi_{i j}+\varphi_{j k}
$$

for each triangle with vertices $1 \leq i<j<k \leq n$ in the triangulation $\Gamma$.

The Grassmannian $\operatorname{Gr}(2, n)$ is obtained as a symplectic reduction of the space $\operatorname{Mat}_{n \times 2}(\mathbb{C}) \cong\left(\mathbb{C}^{2}\right)^{n}$ of $n \times 2$ matrices by the right $U(2)$-action, and hence the Gelfand-MacPherson correspondence GM82 gives an isomorphism between the polygon space and a symplectic reduction of $\operatorname{Gr}(2, n)$ 
by the $\mathbb{T}_{U(n)}$-action. For a later use, we describe the isomorphism explicitly. Since the moment map of of the right $U(2)$-action on $\operatorname{Mat}_{n \times 2}(\mathbb{C})$ is given by

$$
\mu_{U(2)}: \operatorname{Mat}_{n \times 2}(\mathbb{C}) \rightarrow \sqrt{-1} \mathfrak{u}(2), \quad\left(\begin{array}{cc}
z_{1} & w_{1} \\
\vdots & \vdots \\
z_{n} & w_{n}
\end{array}\right) \mapsto \frac{1}{2} \sum_{i=1}^{n}\left(\begin{array}{cc}
\left|z_{i}\right|^{2} & \bar{z}_{i} w_{i} \\
z_{i} \bar{w}_{i} & \left|w_{i}\right|^{2}
\end{array}\right)
$$

the level set $\mu_{U(2)}^{-1}\left(\lambda \mathbf{1}_{2}\right)$ of $\mu_{U(2)}$ consists of $\left(z_{i}, w_{i}\right)_{i} \in \operatorname{Mat}_{n \times 2}(\mathbb{C})$ satisfying

$$
\sum_{i=1}^{n}\left|z_{i}\right|^{2}=\sum_{i=1}^{n}\left|w_{i}\right|^{2}=2 \lambda, \quad \sum_{i=1}^{n} z_{i} \bar{w}_{i}=0,
$$

and hence

$$
Z=\left(z_{i}, w_{i}\right)_{i} \longmapsto \frac{1}{2} Z Z^{*}=\frac{1}{2}\left(z_{i} \overline{z_{j}}+w_{i} \overline{w_{j}}\right)_{i, j}
$$

gives an isomorphism $\mu_{U(2)}^{-1}\left(\lambda \mathbf{1}_{2}\right) / U(2) \rightarrow \mathcal{O}_{\lambda} \cong \operatorname{Gr}(2, n)$. The moment map $\mu_{\mathbb{T}_{U(n)}}: \operatorname{Mat}_{n \times 2}(\mathbb{C}) \rightarrow \mathbb{R}^{n}$ of the left $\mathbb{T}_{U(n)}$-action on $\operatorname{Mat}_{n \times 2}(\mathbb{C})$ is given by

$$
\left(\begin{array}{cc}
z_{1} & w_{1} \\
\vdots & \vdots \\
z_{n} & w_{n}
\end{array}\right) \longmapsto\left(\frac{\left|z_{1}\right|^{2}+\left|w_{1}\right|^{2}}{2}, \ldots, \frac{\left|z_{n}\right|^{2}+\left|w_{n}\right|^{2}}{2}\right)
$$

and thus the projection

$$
\mu_{\mathbb{T}_{U(n)}}^{-1}(2 \boldsymbol{r}) \cong \prod_{i=1}^{n} S^{3}\left(2 \sqrt{r_{i}}\right) \longrightarrow \mathbb{T}_{U(n)} \backslash \mu_{\mathbb{T}_{U(n)}^{-1}}(2 \boldsymbol{r}) \cong \prod_{i=1}^{n} S^{2}\left(r_{i}\right)
$$

is written as

$$
\left(\begin{array}{cc}
z_{1} & w_{1} \\
\vdots & \vdots \\
z_{n} & w_{n}
\end{array}\right) \longmapsto\left(\nu\left(z_{1}, w_{1}\right), \ldots, \nu\left(z_{n}, w_{n}\right)\right)
$$

by using the Hopf fibration

$$
\nu: S^{3}(2 \sqrt{r}) \rightarrow S^{2}(r), \quad(z, w) \mapsto\left(\frac{z \bar{w}}{2}, \frac{|z|^{2}-|w|^{2}}{4}\right),
$$

where we regard $S^{3}(2 \sqrt{r}) \subset \mathbb{C}^{2}$ and $S^{2}(r) \subset \mathbb{C} \times \mathbb{R}$. Since the condition (3.17) implies $\sum_{i} \nu\left(z_{i}, w_{i}\right)=0$, the map (3.21) induces an isomorphism

$$
\begin{aligned}
\mathbb{T}_{U(n)} \|_{2 \boldsymbol{r}} \operatorname{Gr}(2, n) & \cong \mathbb{T}_{U(n)} \backslash\left(\mu_{U(2)}^{-1}\left(\lambda \mathbf{1}_{2}\right) \cap \mu_{\mathbb{T}_{U(n)}}^{-1}(2 \boldsymbol{r})\right) / U(2) \\
& \cong\left(\prod_{i=1}^{n} S^{2}\left(r_{i}\right)\right) / \int_{0} S U(2)=\mathcal{M}_{\boldsymbol{r}}
\end{aligned}
$$

Let $\varphi_{i j}$ also denote the pull-back to $\operatorname{Gr}(2, n)$ of the bending Hamiltonian. 
Proposition 3.5 ([NU14, Proposition 4.6]). Two completely integrable systems $\left(\psi_{i j}\right)_{\epsilon(i, j) \in \operatorname{Prn} \Gamma}$ and $\left(\varphi_{i j}\right)_{\epsilon(i, j) \in \operatorname{Prn} \Gamma}$ are related by

$$
\varphi_{i j}=\psi_{i j}-\frac{1}{2} \sum_{k=i}^{j-1} \psi_{k, k+1},
$$

and thus $\Psi_{\Gamma}$ induces $\Phi_{\Gamma}$ on each polygon space $\mathcal{M}_{\boldsymbol{r}}$ under the symplectic reduction.

Note that $\varphi_{i, i+1}=(1 / 2) \psi_{i, i+1}$ is not an action coordinate, since its Hamiltonian flow has period $\pi(\neq 2 \pi)$. We give a proof of this proposition for readers' convenience.

Proof. We first note that the function $\psi_{i, i+1}$ associated to the leaf $\epsilon(i, i+1)$ is given by

$$
\psi_{i, i+1}\left(\left[z_{k}, w_{k}\right]\right)=\frac{\left|z_{i}\right|^{2}+\left|w_{i}\right|^{2}}{2}, \quad\left[z_{k}, w_{k}\right]_{k} \in \operatorname{Gr}(2, n),
$$

which coincides with $2 \varphi_{i, i+1}\left(\left[z_{k}, w_{k}\right]\right)$. Recall that, for $|i-j| \geq 2$,

$$
\psi_{i j}\left(\left[z_{k}, w_{k}\right]\right)=\lambda_{1}^{(i, j)}\left(\left[z_{k}, w_{k}\right]\right) \geq \lambda_{2}^{(i, j)}\left(\left[z_{k}, w_{k}\right]\right) \geq 0
$$

are the first and second eigenvalues of

$$
\frac{1}{2}\left(\begin{array}{cc}
z_{i} & w_{i} \\
\vdots & \vdots \\
z_{j-1} & w_{j-1}
\end{array}\right)\left(\begin{array}{ccc}
\bar{z}_{i} & \ldots & \bar{z}_{j-1} \\
\bar{w}_{i} & \ldots & \bar{w}_{j-1}
\end{array}\right)
$$

Then the $2 \times 2$ Hermitian matrix

$$
\begin{aligned}
& \frac{1}{2}\left(\begin{array}{ccc}
\bar{z}_{i} & \ldots & \bar{z}_{j-1} \\
\bar{w}_{i} & \ldots & \bar{w}_{j-1}
\end{array}\right)\left(\begin{array}{cc}
z_{i} & w_{i} \\
\vdots & \vdots \\
z_{j-1} & w_{j-1}
\end{array}\right)=\frac{1}{2} \sum_{k=i}^{j-1}\left(\begin{array}{cc}
\left|z_{k}\right|^{2} & \bar{z}_{k} w_{k} \\
z_{k} \bar{w}_{k} & \left|w_{k}\right|^{2}
\end{array}\right) \\
& =\frac{1}{4} \sum_{k=i}^{j-1}\left(\begin{array}{cc}
\left|z_{k}\right|^{2}-\left|w_{k}\right|^{2} & 2 \bar{z}_{k} w_{k} \\
2 z_{k} \bar{w}_{k} & \left|w_{k}\right|^{2}-\left|z_{k}\right|^{2}
\end{array}\right)+\sum_{k=i}^{j-1} \frac{\left|z_{k}\right|^{2}+\left|w_{k}\right|^{2}}{4}\left(\begin{array}{cc}
1 & 0 \\
0 & 1
\end{array}\right)
\end{aligned}
$$

has eigenvalues $\lambda_{1}^{(i, j)} \geq \lambda_{2}^{(i, j)}$. Since

$$
\frac{1}{4} \sum_{k=i}^{j-1}\left(\begin{array}{cc}
\left|z_{k}\right|^{2}-\left|w_{k}\right|^{2} & 2 \bar{z}_{k} w_{k} \\
2 z_{k} \bar{w}_{k} & \left|w_{k}\right|^{2}-\left|z_{k}\right|^{2}
\end{array}\right) \in \sqrt{-1} \mathfrak{s u}(2)
$$


has eigenvalues $\pm\left\|\sum_{k=i}^{j-1} \nu\left(z_{k}, w_{k}\right)\right\|= \pm \varphi_{i j}\left(\left[z_{k}, w_{k}\right]\right)$, we have

$$
\begin{aligned}
& \lambda_{1}^{(i, j)}=\varphi_{i j}+\sum_{k=i}^{j-1} \frac{\left|z_{k}\right|^{2}+\left|w_{k}\right|^{2}}{4}, \\
& \lambda_{2}^{(i, j)}=-\varphi_{i j}+\sum_{k=i}^{j-1} \frac{\left|z_{k}\right|^{2}+\left|w_{k}\right|^{2}}{4},
\end{aligned}
$$

which prove the proposition.

We introduce another coordinate $(u(i, j))_{\epsilon(i, j) \in \operatorname{Prn} \Gamma}$ on $\mathbb{R}^{2 n-4}$ corresponding to $\left(\varphi_{i j}\right)_{\epsilon(i, j) \in \operatorname{Prn} \Gamma}$ defined by

$$
u(i, j)=u_{i j}-\frac{1}{2} \sum_{k=i}^{j-1} u_{k, k+1} .
$$

Corollary 3.6. The moment polytope $\Delta_{\Gamma}=\Psi_{\Gamma}\left(\mathcal{O}_{\lambda}\right)$ is defined by triangle inequalities

$$
|u(i, j)-u(j, k)| \leq u(i, k) \leq u(i, j)+u(j, k)
$$

for each triangle with vertices $1 \leq i<j<k \leq n$ in the triangulation $\Gamma$.

In terms of the action coordinate $\left(u_{i j}\right)_{\epsilon(i, j) \in \operatorname{Prn} \Gamma}$, the inequalities 3.34 are written as

$$
\begin{aligned}
& u_{i k} \geq u_{i j}-u_{j k}+\sum_{l=j}^{k-1} u_{l, l+1}, \\
& u_{i k} \geq u_{j k}-u_{i j}+\sum_{l=i}^{j-1} u_{l, l+1}, \\
& u_{i k} \leq u_{i j}+u_{j k} .
\end{aligned}
$$

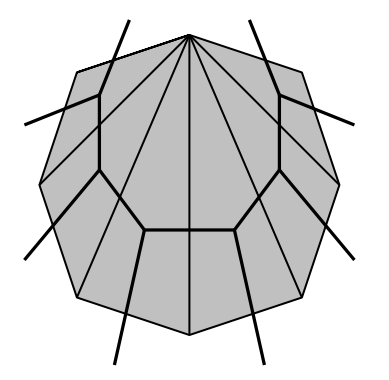

Figure 3.1: The caterpillar 
Example 3.7 (Hausmann and Knutson [HK97]). For the triangulation $\Gamma_{\text {cat }}$ given by

$$
\text { Int } \Gamma_{\text {cat }}:=\{\epsilon(1,3), \epsilon(1,4), \ldots, \epsilon(1, n-2)\}
$$

shown in Figure 3.1 called the caterpillar, the integrable system $\Psi_{\mathrm{GC}}:=$ $\Psi_{\Gamma_{\text {cat }}}$ gives the Gelfand-Cetlin system introduced by Guillemin and Sternberg [GS83, and the triangle inequalities (3.34) give the Gelfand-Cetlin pattern

$$
\begin{aligned}
& \lambda \quad \sum_{i=1}^{n-1} \psi_{i, i+1}-\lambda \\
& \begin{array}{lll}
\lambda \uparrow & \geqslant \\
\psi_{1, n-1} & \sum_{i=1}^{n-2} \psi_{i, i+1}-\psi_{1, n-1}
\end{array} \\
& \uparrow 1, \lambda \\
& \psi_{1, n-2} \quad \sum_{i=1}^{n-3} \psi_{i, i+1}-\psi_{1, n-2}
\end{aligned}
$$

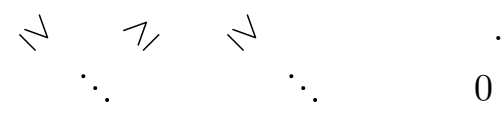

$$
\begin{aligned}
& \lambda \geqslant \geqslant \geqslant \\
& \psi_{13} \quad \sum_{i=1}^{2} \psi_{i, i+1}-\psi_{13} \\
& \checkmark 1 \\
& \psi_{12}
\end{aligned}
$$

Suppose that we have two triangulations $\Gamma, \Gamma^{\prime}$ of $P$ which are related by a Whitehead move in a quadrilateral $P_{0}$ with vertices $1 \leq a<b<c<d \leq n$ (see Figure 3.2, where $P_{0}$ is unshaded). Let $\Gamma^{\prime \prime}$ be the subdivision of $P$

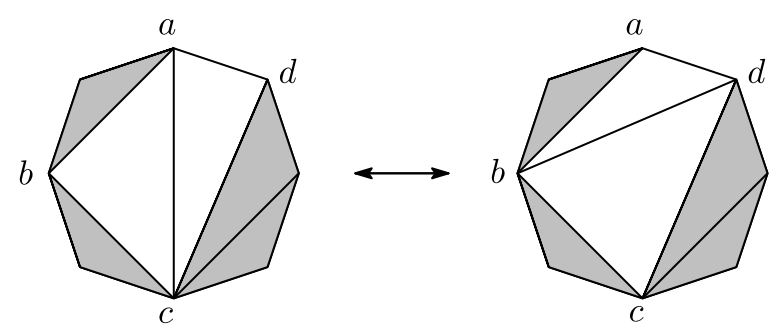

Figure 3.2: A whitehead move

given by common diagonals in $\Gamma$ and $\Gamma^{\prime}$ (see Figure 3.3); its dual graph is obtained from that of $\Gamma$ (resp. $\Gamma^{\prime}$ ) by contracting the edge $\epsilon(a, c) \in \Gamma$ (resp. $\epsilon(b, d) \in \Gamma^{\prime}$ ). Note that the action coordinates on $\Delta_{\Gamma} \subset \mathbb{R}^{\operatorname{Prn} \Gamma}$ and $\Delta_{\Gamma^{\prime}} \subset \mathbb{R}^{\operatorname{Prn} \Gamma^{\prime}}$ are written as

$$
\boldsymbol{u}=\left(\left(u_{i j}\right)_{\epsilon(i, j) \in \operatorname{Prn} \Gamma^{\prime \prime}}, u_{a c}\right), \quad \boldsymbol{u}^{\prime}=\left(\left(u_{i j}\right)_{\epsilon(i, j) \in \operatorname{Prn} \Gamma^{\prime \prime}}, u_{b d}\right),
$$

respectively. 


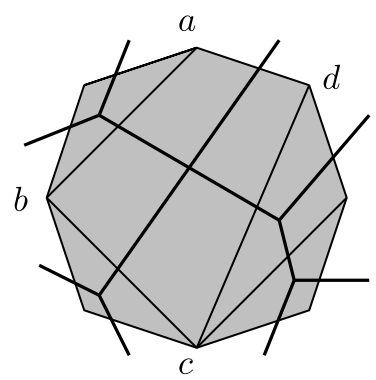

Figure 3.3: A subdivision of $P$ given by common diagonals in two triangulations in Figure 3.2 .

Proposition 3.8. Let $\Gamma, \Gamma^{\prime}$ be two triangulations of $P$ as above. Then the piecewise linear transform $\boldsymbol{u} \mapsto \boldsymbol{u}^{\prime}$ defined by

$u_{b d}=-u_{a c}+u_{a b}+u_{b c}+u_{c d}+u_{a d}-\min \left(u_{a b}+u_{c d}, u_{a d}+u_{b c}-\sum_{i=b}^{c-1} u_{i, i+1}\right)$

gives a bijection between $\Delta_{\Gamma}$ and $\Delta_{\Gamma^{\prime}}$.

Remark 3.9. The piecewise linear transformation (3.41) is different from the one given in [NU14, Proposition 3.5].

Proof. For each fixed $\left(\varphi_{a b}, \varphi_{b c}, \varphi_{c d}, \varphi_{a d}\right)=\left(r_{1}, r_{2}, r_{3}, r_{4}\right) \in\left(\mathbb{R}^{>0}\right)^{4}$, the ranges of the bending Hamiltonians $\varphi_{a c}$ and $\varphi_{b d}$ are given by

$$
\begin{aligned}
& \max \left\{\left|r_{1}-r_{2}\right|,\left|r_{3}-r_{4}\right|\right\} \leq u(a, c) \leq \min \left\{r_{1}+r_{2}, r_{3}+r_{4}\right\}, \\
& \max \left\{\left|r_{1}-r_{4}\right|,\left|r_{2}-r_{3}\right|\right\} \leq u(b, d) \leq \min \left\{r_{1}+r_{4}, r_{2}+r_{3}\right\},
\end{aligned}
$$

respectively. Since

$$
\begin{aligned}
- & \max \left\{\left|r_{1}-r_{2}\right|,\left|r_{3}-r_{4}\right|\right\} \\
& =\min \left\{\min \left\{r_{1}-r_{2}, r_{3}-r_{4}\right\}, \min \left\{r_{2}-r_{1}, r_{4}-r_{3}\right\}\right\} \\
& =\min \left\{\min \left\{r_{1}+r_{4}, r_{2}+r_{3}\right\}-r_{2}-r_{4}, \min \left\{r_{1}+r_{4}, r_{2}+r_{3}\right\}-r_{1}-r_{3}\right\} \\
& =\min \left\{r_{1}+r_{4}, r_{2}+r_{3}\right\}+\min \left\{-r_{2}-r_{4},-r_{1}-r_{3}\right\} \\
& =\min \left\{r_{1}+r_{4}, r_{2}+r_{3}\right\}+\min \left\{r_{1}+r_{3}, r_{2}+r_{4}\right\}-\left(r_{1}+r_{2}+r_{3}+r_{4}\right),
\end{aligned}
$$

the lengths of the ranges are the same;

$$
\begin{aligned}
\min \left\{r_{1}+r_{2}, r_{3}+r_{4}\right\}-\max \left\{\left|r_{1}-r_{2}\right|,\left|r_{3}-r_{4}\right|\right\} \\
=\min \left\{r_{1}+r_{4}, r_{2}+r_{3}\right\}-\max \left\{\left|r_{1}-r_{4}\right|,\left|r_{2}-r_{3}\right|\right\} .
\end{aligned}
$$


Thus the map $u(a, c) \mapsto u(b, d)$ defined by

$$
\begin{aligned}
u(b, d) & =-u(a, c)+\min \left\{r_{1}+r_{2}, r_{3}+r_{4}\right\}+\max \left\{\left|r_{1}-r_{4}\right|,\left|r_{2}-r_{3}\right|\right\} \\
& =-u(a, c)+r_{1}+r_{2}+r_{3}+r_{4}-\min \left\{r_{1}+r_{3}, r_{2}+r_{4}\right\}
\end{aligned}
$$

gives a bijection between the ranges of $\varphi_{a c}$ and $\varphi_{b d}$. One can easily check that this map is written as (3.41) under the coordinate change (3.33).

\section{Degenerations of Grassmannians}

Speyer and Sturmfels [SS04] have shown that toric degenerations of the Grassmannian $\operatorname{Gr}(2, n)$ are parametrized by the tropical Grassmannian, and its top dimensional cells are in one-to-one correspondence with the set of trivalent trees $\Gamma$ with $n$-leaves. For each $\Gamma$, the corresponding toric degeneration $f_{\Gamma}: \mathfrak{X}_{\Gamma} \rightarrow \mathbb{C}^{n-3}$ of $\operatorname{Gr}(2, n)$ can be constructed as follows. Let $\boldsymbol{p}=\left[p_{i j}\right]_{1 \leq i<j \leq n}$ be a homogeneous coordinate on $\mathbb{P}\left(\bigwedge^{2} \mathbb{C}^{n}\right)$ so that $\operatorname{Gr}(2, n) \subset \mathbb{P}\left(\bigwedge^{\overline{2}} \mathbb{C}^{n}\right)$ is given by the Plücker relations

$$
F_{i j k l}(\boldsymbol{p})=p_{i j} p_{k l}-p_{i k} p_{j l}+p_{i l} p_{j k}=0
$$

for $1 \leq i<j<k<l \leq n$ | For each $1 \leq i, j \leq n$, let $\gamma(i, j)$ denote the path in the tree $\Gamma$ connecting the $i$-th and $j$-th leaves $\epsilon(i, i+1), \epsilon(j, j+1)$. We

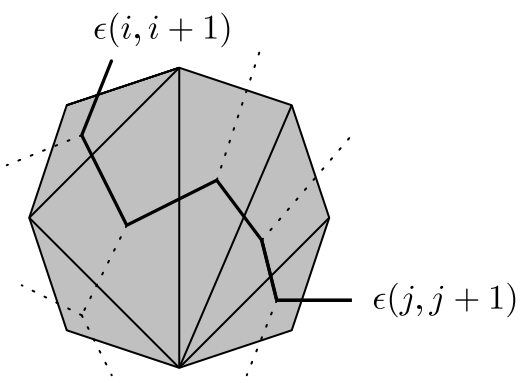

Figure 4.1: A path $\gamma(i, j)$ connecting the $i$-th and $j$-th leaves

introduce $n-3$ deformation parameters $\boldsymbol{t}=\left(t_{k l}\right)_{\epsilon(k, l) \in \operatorname{Int} \Gamma} \in \mathbb{C}^{\operatorname{Int} \Gamma}$. Define a weight $\boldsymbol{w}(i, j)=\left(w_{k l}(i, j)\right)_{\epsilon(k, l) \in \operatorname{Int} \Gamma} \in \mathbb{Q}^{\operatorname{Int} \Gamma}$ of a Plücker coordinate $p_{i j}$ by

$$
w_{k l}(i, j)= \begin{cases}1 / 2 & \text { if } \gamma(i, j) \text { contains the edge } \epsilon(k, l), \\ 0 & \text { otherwise }\end{cases}
$$

\footnotetext{
${ }^{1}$ Note that the indices of the the Plücker coordinates are labels of leaves of $\Gamma$ (or equivalently, sides of the reference polygon $P$ ), while the indices of the Hamiltonians $\psi_{i j}$ (and hence, those of coordinates on the SYZ mirror) are labels of vertices of $P$.
} 
and consider an action of $\left(\mathbb{C}^{\times}\right)^{\operatorname{Int} \Gamma}$ on $\bigwedge^{2} \mathbb{C}^{n}$ given by

$$
\boldsymbol{t} \cdot \boldsymbol{p}=\left(\boldsymbol{t}^{\boldsymbol{w}(i, j)} p_{i j}\right)=\left(\prod_{\epsilon(k, l)} t_{k l}^{w_{k l}(i, j)} p_{i j}\right)=\left(\prod_{\epsilon(k, l) \subset \gamma(i, j)} t_{k l}^{1 / 2} p_{i j}\right) .
$$

For a polynomial

$$
F(\boldsymbol{p})=\sum_{I=\left(i_{1}, j_{1}, \ldots, i_{m}, j_{m}\right)} c_{I} p_{i_{1} j_{1}} \ldots p_{i_{m} j_{m}}
$$

in Plücker coordinates, let

$$
w_{k l}(F)=\max \left\{w_{k l}\left(i_{1}, j_{1}\right)+\cdots+w_{k l}\left(i_{m}, j_{m}\right) \mid c_{I} \neq 0\right\}
$$

be the maximum of weights of monomials in $F(\boldsymbol{p})$ with respect to $t_{k l}$, and define $F^{\Gamma}(\boldsymbol{p}, \boldsymbol{t}) \in \mathbb{C}[\boldsymbol{p}, \boldsymbol{t}]$ by

$$
F^{\Gamma}(\boldsymbol{p}, \boldsymbol{t})=\boldsymbol{t}^{\boldsymbol{w}(F)} F\left(\boldsymbol{t}^{-\boldsymbol{w}(i, j)} p_{i j}\right),
$$

where

$$
\boldsymbol{t}^{\boldsymbol{w}(F)}=\prod_{\epsilon(k, l) \in \operatorname{Int} \Gamma} t_{k l}^{w_{k l}(F)} .
$$

Then the degenerating family $f_{\Gamma}: \mathfrak{X}_{\Gamma} \rightarrow \mathbb{C}^{n-3}$ associated with $\Gamma$ is given by

$$
\mathfrak{X}_{\Gamma}=\left\{(\boldsymbol{p}, \boldsymbol{t}) \in \mathbb{P}\left(\bigwedge^{2} \mathbb{C}^{n}\right) \times \mathbb{C}^{n-3} \mid F_{i j k l}^{\Gamma}(\boldsymbol{p}, \boldsymbol{t})=0, i<j<k<l\right\},
$$

whose central fiber $X_{\Gamma}=f_{\Gamma}^{-1}(0, \ldots, 0)$ is a toric variety with moment polytope $\Delta_{\Gamma}$ (see [NU14, Example 5.2] for the case $n=5$ ). [NU14, Theorem 1.2] combined with [HK15, Theorem 5.4] gives the following.

Theorem 4.1. The completely integrable system $\Psi_{\Gamma}$ on $\operatorname{Gr}(2, n)$ can be deformed into a toric moment map $\mu_{\mathbb{T}_{\Gamma}}$ on $X_{\Gamma}$ with moment polytope $\Delta_{\Gamma}$. There exists a map $\phi: \operatorname{Gr}(2, n) \rightarrow X_{\Gamma}$ which sends each Lagrangian torus fiber $L_{\Gamma}(\boldsymbol{u})=\Psi_{\Gamma}^{-1}(\boldsymbol{u})$ diffeomorphically to the fiber $\mu_{\mathbb{T}_{\Gamma}}^{-1}(\boldsymbol{u})$ of $\mu_{\mathbb{T}_{\Gamma}}$ over the same point $\boldsymbol{u} \in \operatorname{Int} \Delta_{\Gamma}$.

The map $\phi: \operatorname{Gr}(2, n) \rightarrow X_{\Gamma}$ is given by the gradient-Hamiltonian flow for $f_{\Gamma}$ introduced by Ruan [Rua01]. Harada and Kaveh [HK15, Theorem 5.4] show that the gradient-Hamiltonian flow extends to singular loci of $X_{\Gamma}$.

Let $(X, \omega)$ be a symplectic manifold, and fix a compatible almost complex structure $J$. For a Lagrangian submanifold $L$ in $X$ and a relative homotopy class $\beta \in \pi_{2}(X, L)$, let $\overline{\mathcal{M}}_{1}(X, L ; \beta)$ denote the the moduli space of stable $J$-holomorphic maps of degree $\beta$ from a bordered Riemann surface of genus zero with one marked point and with Lagrangian boundary condition. Fix $\boldsymbol{t} \in\left(\mathbb{C}^{\times}\right)^{n-3} \subset \mathbb{C}^{n-3}$ sufficiently close to the origin, and let $J_{\boldsymbol{t}}$ be the complex structure on the fiber $X_{\boldsymbol{t}}=f_{\Gamma}^{-1}(\boldsymbol{t})$ of the family 4.8). By using a 
symplectomorphism $\operatorname{Gr}(2, n) \rightarrow X_{t}$ given by the gradient-Hamiltonian flow, we regard $J_{\boldsymbol{t}}$ as a complex structure on $\operatorname{Gr}(2, n)$. The fact that the toric variety $X_{\Gamma}$ is Fano and admits a small resolution [NU14, Section 8] enable us to apply the argument in [NNU10, Section 9] to obtain the following:

Theorem 4.2 ([NNU10, Proposition 9.16]). For each $\boldsymbol{u} \in \operatorname{Int} \Delta_{\Gamma}$ and $\beta \in$ $\pi_{2}\left(\operatorname{Gr}(2, n), L_{\Gamma}(\boldsymbol{u})\right)$ of Maslov index two, there exists a diffeomorphism

$$
\overline{\mathcal{M}}_{1}\left(\operatorname{Gr}(2, n), L_{\Gamma}(\boldsymbol{u}) ; \beta\right) \stackrel{\sim}{\rightarrow} \overline{\mathcal{M}}_{1}\left(X_{\Gamma}, \mu_{\mathbb{T}_{\Gamma}}^{-1}(\boldsymbol{u}) ; \beta\right)
$$

such that

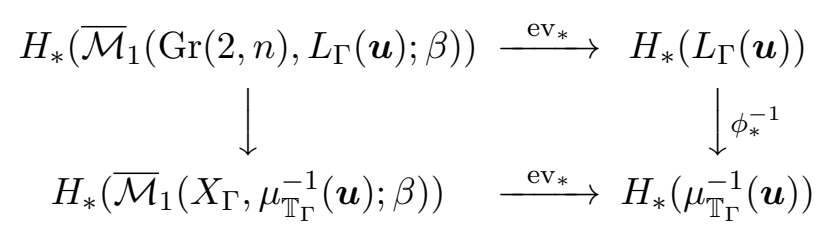

commutes, where ev: $\overline{\mathcal{M}}_{1}(X, L ; \beta) \rightarrow L$ is the evaluation map at boundary marked points, and $\beta$ in the right hand side of (4.9) is regarded as a homotopy class in $X_{\Gamma}$ via the isomorphism $\phi_{*}: \pi_{2}\left(\operatorname{Gr}(2, n), L_{\Gamma}(\boldsymbol{u})\right) \rightarrow$ $\pi_{2}\left(X_{\Gamma}, \mu_{\mathbb{T}_{\Gamma}}^{-1}(\boldsymbol{u})\right)$.

Suppose that we have two triangulations $\Gamma$ and $\Gamma^{\prime}$ related by a Whitehead move as in Figure 3.2 . Then the subdivision $\Gamma^{\prime \prime}$ defined by common diagonals in $\Gamma$ and $\Gamma^{\prime}$ gives a subfamily

$$
f_{\Gamma^{\prime \prime}}: \mathfrak{X}_{\Gamma^{\prime \prime}} \longrightarrow \mathbb{C}^{n-4}=\mathbb{C}^{\operatorname{Int} \Gamma^{\prime \prime}}
$$

of $f_{\Gamma}: \mathfrak{X}_{\Gamma} \rightarrow \mathbb{C}^{\operatorname{Int} \Gamma}$ (resp. $f_{\Gamma^{\prime}}: \mathfrak{X}_{\Gamma^{\prime}} \rightarrow \mathbb{C}^{\operatorname{Int} \Gamma^{\prime}}$ ) defined by $t_{a c}=1$ (resp. $\left.t_{b d}=1\right)$, on which $\Psi_{\Gamma}=\left(\psi_{i j}\right)_{\epsilon(i, j) \in \operatorname{Prn} \Gamma}$ (resp. $\left.\Psi_{\Gamma^{\prime}}=\left(\psi_{i j}\right)_{\epsilon(i, j) \in \operatorname{Prn} \Gamma^{\prime}}\right)$ can be deformed into a completely integrable system $\Psi_{\Gamma}^{0}=\left(\psi_{i j}^{0}\right)_{\epsilon(i, j) \in \operatorname{Prn} \Gamma}$ (resp. $\left.\Psi_{\Gamma^{\prime}}=\left(\psi_{i j}^{0}\right)_{\epsilon(i, j) \in \operatorname{Prn} \Gamma^{\prime}}\right)$ on the central fiber $X_{0}=f_{\Gamma^{\prime \prime}}^{-1}(0, \ldots, 0)$. We write fibers of $\Psi_{\Gamma}^{0}$ as $L_{\Gamma}^{0}(\boldsymbol{u})=\left(\Psi_{\Gamma}^{0}\right)^{-1}(\boldsymbol{u})$.

Corollary 4.3. For each $\boldsymbol{u} \in \operatorname{Int} \Delta_{\Gamma}$ and $\beta \in \pi_{2}\left(\operatorname{Gr}(2, n), L_{\Gamma}(\boldsymbol{u})\right)$ of Maslov index two, there exists a diffeomorphism

$$
\overline{\mathcal{M}}_{1}\left(\operatorname{Gr}(2, n), L_{\Gamma}(\boldsymbol{u}) ; \beta\right) \stackrel{\sim}{\rightarrow} \overline{\mathcal{M}}_{1}\left(X_{0}, L_{\Gamma}^{0}(\boldsymbol{u}) ; \beta\right)
$$

which commutes with evaluation maps on the homology groups. The same is true for $\Gamma^{\prime}$.

We observe defining equations for $X_{0}$ are:

$$
F_{i j k l}^{\Gamma^{\prime \prime}}(\boldsymbol{p}, \mathbf{0})=F_{i j k l}^{\Gamma}\left(\boldsymbol{p},\left(0, \ldots, 0, t_{a c}=1\right)\right)=0 .
$$


If the paths $\gamma(i, k)$ and $\gamma(j, l)$ intersect transversally in the interior of the quadrilateral $P_{0}$ as in Figure 4.2 , then all monomials in $F_{i j k l}$ have the same weight, and thus the Plücker relation is unchanged:

$$
F_{i j k l}^{\Gamma^{\prime \prime}}(\boldsymbol{p}, \mathbf{0})=F_{i j k l}(\boldsymbol{p})=p_{i j} p_{k l}-p_{i k} p_{j l}+p_{i l} p_{j k} .
$$

In the case where $\gamma(i, k)$ and $\gamma(j, l)$ share at least one interior edge, the Plücker relation is deformed into a binomial

$$
F_{i j k l}^{\Gamma^{\prime \prime}}(\boldsymbol{p}, \mathbf{0})=-p_{i k} p_{j l}+p_{i l} p_{j k},
$$

where we assume that $\gamma(i, j)$ and $\gamma(k, l)$ do not share any edge in $\Gamma^{\prime \prime}$ (see Figure 4.3).

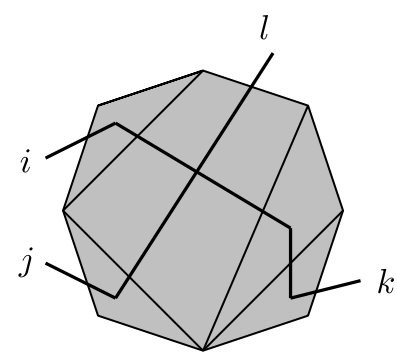

Figure 4.2: $\gamma(i, k)$ and $\gamma(j, l)$ intersect transversally.

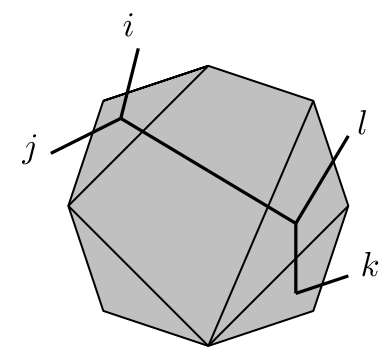

Figure 4.3: $\gamma(i, k)$ and $\gamma(j, l)$ share an interior edge in $\Gamma^{\prime \prime}$.

We give a description of $X_{0}$ following an idea in HMM11. By cutting the reference polygon $P$ along the diagonals in $\Gamma^{\prime \prime}$, we obtain a subdivision of $P$ into one quadrilateral $P_{0}$ and $n-4$ triangles $P_{1}, \ldots, P_{n-4}$, and the corresponding forest (i.e., a set of trees) $\Gamma_{0}, \Gamma_{1}, \ldots, \Gamma_{n-4}$. For each subpolygon

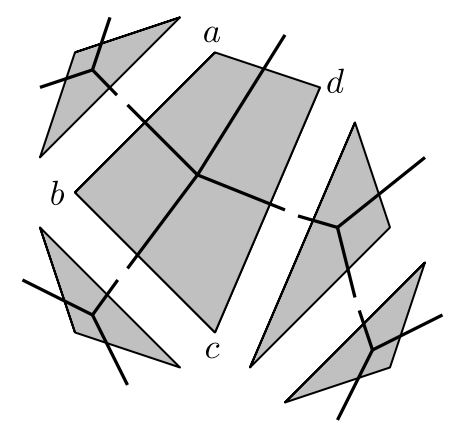

Figure 4.4: A subdivision of $P$ and the corresponding forest.

$P_{\alpha}$, we associate a cone $\widetilde{\operatorname{Gr}}\left(P_{\alpha}\right) \subset \bigwedge^{2} \mathbb{C}^{\Gamma_{\alpha}}$ over the Grassmannian

$$
\operatorname{Gr}\left(P_{\alpha}\right):=\operatorname{Gr}\left(2, \mathbb{C}^{\Gamma_{\alpha}}\right) \cong \begin{cases}\operatorname{Gr}(2,4) & \alpha=0 \\ \mathbb{P}^{2} & \alpha=1, \ldots, n-4\end{cases}
$$


on which the Plücker coordinates $\left(p_{\epsilon, \epsilon^{\prime}}^{P_{\alpha}}\right)_{\epsilon, \epsilon^{\prime} \in \Gamma_{\alpha}}$ are indexed by pairs of (boundary) edges of $\Gamma_{\alpha}$. We consider an action of a torus $\left(\mathbb{C}^{\times}\right)^{\amalg_{\alpha} \Gamma_{\alpha}} \cong\left(\mathbb{C}^{\times}\right)^{3 n-8}$ on $\prod_{\alpha=0}^{n-4} \widetilde{\operatorname{Gr}}\left(P_{\alpha}\right)$ defined by

$$
\boldsymbol{\tau} \cdot\left(p_{\epsilon, \epsilon^{\prime}}^{P_{\alpha}}\right)_{\epsilon, \epsilon^{\prime}}=\left(\tau_{\epsilon} \tau_{\epsilon^{\prime}} p_{\epsilon, \epsilon^{\prime}}^{P_{\alpha}}\right)_{\epsilon, \epsilon^{\prime}}
$$

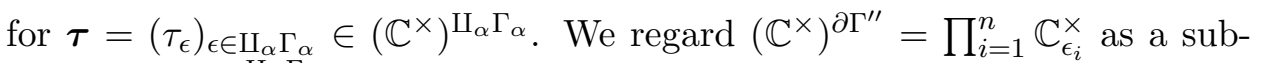
group of $\left(\mathbb{C}^{\times}\right)^{\amalg_{\alpha} \Gamma_{\alpha}}$ by identifying leaves $\epsilon_{i}=\epsilon(i, i+1)$ of $\Gamma^{\prime \prime}$ with corresponding edges in the forest $\amalg_{\alpha} \Gamma_{\alpha}$, and define $\mathbb{C}_{\partial P}^{\times} \cong \mathbb{C}^{\times}$to be the diagonal subgroup of $\left(\mathbb{C}^{\times}\right)^{\partial \Gamma^{\prime \prime}}\left(\subset\left(\mathbb{C}^{\times}\right)^{\amalg_{\alpha} \Gamma_{\alpha}}\right)$. For an interior edge $\epsilon=\epsilon(i, j) \in \operatorname{Int} \Gamma^{\prime \prime}$, let $\epsilon^{+}, \epsilon^{-}$be two copies of $\epsilon$ in $\amalg_{\alpha} \Gamma_{\alpha}$, and define

$$
\mathbb{C}_{\epsilon^{+}, \epsilon^{-}}^{\times}=\left\{\left(\tau, \tau^{-1}\right) \in \mathbb{C}_{\epsilon^{+}}^{\times} \times \mathbb{C}_{\epsilon^{-}}^{\times} \mid \tau \in \mathbb{C}^{\times}\right\} \cong \mathbb{C}^{\times}
$$

to be the anti-diagonal subgroup of $\mathbb{C}_{\epsilon^{+}}^{\times} \times \mathbb{C}_{\epsilon^{-}}^{\times}\left(\subset\left(\mathbb{C}^{\times}\right)^{\amalg_{\alpha} \Gamma_{\alpha}}\right)$. Then the torus action (4.17) induces an action of the $(n-3)$-dimensional subtorus of $\left(\mathbb{C}^{\times}\right)^{\amalg_{\alpha} \Gamma_{\alpha}}$

$$
\mathbb{C}_{\partial P}^{\times} \times \prod_{\epsilon \in \operatorname{Int} \Gamma^{\prime \prime}} \mathbb{C}_{\epsilon^{+}, \epsilon^{-}}^{\times} \cong \mathbb{C}^{\times} \times\left(\mathbb{C}^{\times}\right)^{n-4}
$$

on $\prod_{\alpha} \widetilde{\operatorname{Gr}}\left(P_{\alpha}\right)$. We define

$$
\mathbb{T}_{\Gamma^{\prime \prime}}^{\mathbb{C}}=\left(\mathbb{C}^{\times}\right)^{\amalg_{\alpha} \Gamma_{\alpha}} / \prod_{\epsilon \in \operatorname{Int} \Gamma^{\prime \prime}} \mathbb{C}_{\epsilon^{+}, \epsilon^{-}}^{\times} \cong\left(\mathbb{C}^{\times}\right)^{\Gamma^{\prime \prime}} \cong\left(\mathbb{C}^{\times}\right)^{2 n-4} .
$$

Proposition 4.4. The central fiber $X_{0}$ of the family 4.11) is given by the GIT quotient

$$
X_{0} \cong \prod_{\alpha=0}^{n-4} \widetilde{\operatorname{Gr}}\left(P_{\alpha}\right) / /\left(\mathbb{C}_{\partial P}^{\times} \times \prod_{\epsilon \in \operatorname{Int} \Gamma^{\prime \prime}} \mathbb{C}_{\epsilon^{+}, \epsilon^{-}}^{\times}\right),
$$

and the inclusion $X_{0} \hookrightarrow \mathbb{P}\left(\bigwedge^{2} \mathbb{C}^{n}\right)$ is given by

$$
p_{i j}=\prod_{\epsilon, \epsilon^{\prime} \subset \gamma(i, j)} p_{\epsilon, \epsilon^{\prime}}^{P_{\alpha}}
$$

where the product in the right hand side is taken over a sequence of edges of $\amalg_{\alpha} \Gamma_{\alpha}$ contained in the path $\gamma(i, j)$. Furthermore, the induced action of $\mathbb{T}_{\Gamma^{\prime \prime}}^{\mathbb{C}} / \mathbb{C}_{\partial P}^{\times} \cong\left(\mathbb{C}^{\times}\right)^{2 n-5}$ on $X_{0}$ is the complexification of the Hamiltonian torus

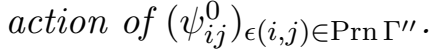

See [NU14, Section 5, 6] for more detail. Note that the $\left(\mathbb{C}^{\times}\right)^{\partial \Gamma^{\prime \prime}}$-action on $X_{0}$ coincides with the complexification of the $\mathbb{T}_{U(n)}$-action. Define a subgroup $\mathbb{T}_{\Gamma^{\prime \prime} \backslash \Gamma_{0}}^{\mathbb{C}} \cong\left(\mathbb{C}^{\times}\right)^{2 n-8}$ of $\mathbb{T}_{\Gamma^{\prime \prime}}^{\mathbb{C}} \cong\left(\mathbb{C}^{\times}\right)^{\Gamma^{\prime \prime}}$ by

$$
\begin{aligned}
\mathbb{T}_{\Gamma^{\prime \prime} \backslash \Gamma_{0}}^{\mathbb{C}} & =\left\{\left(\tau_{\epsilon}\right)_{\epsilon \in \Gamma^{\prime \prime}} \mid \tau_{\epsilon}=1 \text { for } \epsilon=\epsilon(a, b), \epsilon(b, c), \epsilon(c, d), \epsilon(a, d)\right\} \\
& \cong\left(\mathbb{C}^{\times}\right)^{\Gamma^{\prime \prime} \backslash\{\epsilon(a, b), \epsilon(b, c), \epsilon(c, d), \epsilon(a, d)\}}
\end{aligned}
$$


and set

$$
\mathbb{T}_{\Gamma_{0}}^{\mathbb{C}}=\mathbb{T}_{\Gamma^{\prime \prime}}^{\mathbb{C}} / \mathbb{T}_{\Gamma^{\prime \prime} \backslash \Gamma_{0}}^{\mathbb{C}} \cong\left(\mathbb{C}^{\times}\right)^{4}
$$

We consider an anti-canonical divisor of $X_{0}$ given by

$$
D_{0}=\bigcup_{i=1}^{n}\left\{p_{i, i+1}=0\right\} \text {. }
$$

For each $\alpha=0, \ldots, n-4$, we define

$$
\widetilde{\mathrm{Gr}}^{\circ}\left(P_{\alpha}\right)=\left\{\left[p_{\epsilon, \epsilon^{\prime}}^{P_{\alpha}}\right] \in \widetilde{\mathrm{Gr}}\left(P_{\alpha}\right) \mid p_{\epsilon, \epsilon^{\prime}}^{P_{\alpha}} \neq 0 \text { for adjacent leaves } \epsilon, \epsilon^{\prime}\right\}
$$

so that its projection image $\operatorname{Gr}^{\circ}\left(P_{\alpha}\right)$ in $\operatorname{Gr}\left(P_{\alpha}\right)$ is a complement of an anticanonical divisor in $\operatorname{Gr}\left(P_{\alpha}\right)$. Since $\widetilde{\operatorname{Gr}}^{\circ}\left(P_{\alpha}\right) \cong\left(\mathbb{C}^{\times}\right)^{3}$ for $\alpha=1, \ldots, n-4$, the torus $\left(\mathbb{C}^{\times}\right)^{\amalg_{\alpha=0}^{n-4} \Gamma_{\alpha}}$ acts freely on $\prod_{\alpha=0}^{n-4} \widetilde{G r}^{\circ}\left(P_{\alpha}\right)$.

Proposition 4.5. The complement $X_{0} \backslash D_{0}$ is isomorphic to the geometric quotient

$$
X_{0} \backslash D_{0} \cong \prod_{\alpha=0}^{n-4} \widetilde{\operatorname{Gr}}^{\circ}\left(P_{\alpha}\right) /\left(\mathbb{C}_{\partial P}^{\times} \times \prod_{\epsilon \in \operatorname{Int} \Gamma^{\prime \prime}} \mathbb{C}_{\epsilon^{+}, \epsilon^{-}}^{\times}\right)
$$

of $\prod_{\alpha} \widetilde{\mathrm{Gr}}^{\circ}\left(P_{\alpha}\right)$.

Proof. First note that the image in $X_{0} \subset \mathbb{P}\left(\bigwedge^{2} \mathbb{C}^{n}\right)$ of the right hand side of 4.28 is the complement of a subvariety in $X_{0}$ defined by $p_{i j}=0$ for any $i<j$ such that $\gamma(i, j)$ contains no path $\epsilon(a, b) \cup \epsilon(c, d), \epsilon(a, d) \cup \epsilon(b, c)$ in $\Gamma_{0}$ connecting opposite sides of $P_{0}$. Since each path $\gamma(i, i+1)$ connecting adjacent leaves contains neither $\epsilon(a, b) \cup \epsilon(c, d)$ nor $\epsilon(a, d) \cup \epsilon(b, c)$, we have $p_{i, i+1}=\prod p_{\epsilon, \epsilon^{\prime}}^{P_{\alpha}} \neq 0$ for $i=1, \ldots, n$, which means that the complement $X_{0} \backslash$ $D_{0}$ contains the right hand side of 4.28 . To show the converse, we renumber $P_{1}, \ldots, P_{n-4}$ if necessary in such a away that, for each $\alpha=1, \ldots, n-4$, the $\alpha$-th triangle $P_{\alpha}$ shares two sides with the polygon $P \backslash\left(P_{1} \cup \cdots \cup P_{\alpha-1}\right)$ with $n-(\alpha-1)$ sides. Suppose that $\Gamma_{1}$ contains the $i$-th and $(i+1)$-st leaves of $\Gamma^{\prime \prime}$. Then the paths $\gamma(i-1, i)$ and $\gamma(i+1, i+2)$ share at least one interior

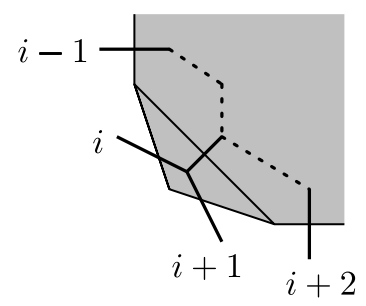

Figure 4.5: Paths connecting adjacent leaves

edge (see Figure 4.5), which implies from (4.15) that the defining equation $F_{i-1, i, i+1, i+2}^{\Gamma^{\prime \prime}}(\boldsymbol{p}, \mathbf{0})=0$ is a binomial

$$
p_{i-1, i} p_{i+1, i+2}=p_{i-1, i+1} p_{i, i+2},
$$


and consequently we obtain $p_{i-1, i+1}, p_{i, i+2} \neq 0$. In other words, we have $p_{j k} \neq 0$ for each pair $(j, k)$ such that the path $\gamma(j, k)$ induces one in $\Gamma^{\prime \prime} \backslash \Gamma_{1}$ connecting adjacent leaves in the polygon $P \backslash P_{1}$ with $n-1$ sides. By repeating this process inductively, we obtain (4.28).

The $\mathbb{T}_{\Gamma^{\prime \prime}}^{\mathbb{C}}$-action on the quotient $X_{0} \backslash D_{0}$ is free, and the projection $\prod_{\alpha=0}^{n-4} \widetilde{\operatorname{Gr}}^{\circ}\left(P_{\alpha}\right) \rightarrow \widetilde{\mathrm{Gr}}^{\circ}\left(P_{0}\right)$ induces the quotient

$$
X_{0} \backslash D_{0} \longrightarrow\left(X_{0} \backslash D_{0}\right) / \mathbb{T}_{\Gamma^{\prime \prime}}^{\mathbb{C}} \cong \operatorname{Gr}^{\circ}\left(P_{0}\right) / \mathbb{T}_{\Gamma_{0}}^{\mathbb{C}} \cong \mathbb{C}^{\times}
$$

which extends to the GIT quotient

$$
X_{0}^{\mathrm{ss}} \longrightarrow X_{0} / / \mathbb{T}_{\Gamma^{\prime \prime}}^{\mathbb{C}} \cong \operatorname{Gr}\left(P_{0}\right) / / \mathbb{T}_{\Gamma_{0}}^{\mathbb{C}} \cong \mathbb{P}^{1} .
$$

Note that the functions $\psi_{a c}^{0}, \psi_{b d}^{0}$ descend to bending Hamiltonians $\varphi_{a c}, \varphi_{b d}$ (up to additive constants) on a 1-dimensional polygon space $\operatorname{Gr}\left(P_{0}\right) / / \mathbb{T}_{\Gamma_{0}}^{\mathbb{C}} \cong$ $\operatorname{Gr}\left(P_{0}\right) / / r \mathbb{T}_{\Gamma_{0}}$ parameterizing spatial quadrilaterals. It follows from Proposition 4.5 that the GIT quotient of $X_{0}$ by the action of the subtorus $\mathbb{T}_{\Gamma^{\prime \prime}}^{\mathbb{C}} \backslash \Gamma_{0}$ induces a torus bundle

$$
X_{0} \backslash D_{0} \longrightarrow\left(X_{0} \backslash D_{0}\right) / \mathbb{T}_{\Gamma^{\prime \prime} \backslash \Gamma_{0}}^{\mathbb{C}} \cong \operatorname{Gr}^{\circ}\left(P_{0}\right)
$$

over $\operatorname{Gr}^{\circ}\left(P_{0}\right)$. The inclusion $\widetilde{\operatorname{Gr}^{\circ}}\left(P_{0}\right) \hookrightarrow \prod_{\alpha} \widetilde{\operatorname{Gr}}^{\circ}\left(P_{\alpha}\right)$ defined by $p_{\epsilon, \epsilon^{\prime}}^{P_{\alpha}}=1$ for all $\alpha \neq 0$ and $\epsilon, \epsilon^{\prime}$ induces a section $\operatorname{Gr}^{\circ}\left(P_{0}\right) \rightarrow X_{0} \backslash D_{0}$ of the torus bundle (4.32), and thus we obtain the following.

Corollary 4.6. The complement $X_{0} \backslash D_{0}$ of the anti-canonical divisor $D_{0}$ is isomorphic to $\operatorname{Gr}^{\circ}\left(P_{0}\right) \times \mathbb{T}_{\Gamma^{\prime \prime}}^{\mathbb{C}} \Gamma_{0} \cong \operatorname{Gr}^{\circ}(2,4) \times\left(\mathbb{C}^{\times}\right)^{2(n-4)}$.

Remark 4.7. From the argument in [NNU10, Section 5], the central fiber $X_{0}$ admits a small resolution $\pi: \widetilde{X}_{0} \rightarrow X_{0}$ such that $\widetilde{X}_{0}$ is a tower of projective planes over $\operatorname{Gr}\left(P_{0}\right)$. The map $\pi$ is isomorphism on $X_{0} \backslash D_{0}$, and the torus bundle structure 4.32 is given by restricting the tower structure to the open subset $X_{0} \backslash D_{0} \subset X_{0}$.

\section{Potential functions}

Let $(X, \omega)$ be a symplectic manifold, and fix a compatible almost complex structure $J$. For a (relatively) spin Lagrangian submanifold $L$ the cohomology group $H^{*}\left(L ; \Lambda_{0}\right)$ has a structure of a filtered $A_{\infty}$-algebra [FOOO09]

$$
\mathfrak{m}_{k}: H^{*}\left(L ; \Lambda_{0}\right)^{\otimes k} \longrightarrow H^{*}\left(L ; \Lambda_{0}\right), \quad k=0,1,2, \ldots
$$

over the Novikov ring

$$
\Lambda_{0}=\left\{\sum_{i=0}^{\infty} a_{i} T^{\lambda_{i}} \mid a_{i} \in \mathbb{C}, \lambda_{i} \in \mathbb{R}_{\geq 0}, \lim _{i \rightarrow \infty} \lambda_{i}=\infty\right\}
$$


defined by 'counting' $J$-holomorphic disks $\left(D^{2}, \partial D^{2}\right) \rightarrow(X, L)$. A solution to the Maurer-Cartan equation

$$
\sum_{k=0}^{\infty} \mathfrak{m}_{k}(b, \ldots, b) \equiv 0 \bmod \operatorname{PD}([L])
$$

is called a weak bounding cochain, where $\mathrm{PD}([L])$ is the Poincaré dual of the fundamental class $[L]$. The potential function is a map $\mathfrak{P}: \mathcal{M}(L) \rightarrow \Lambda_{0}$ from the space $\mathcal{M}(L)$ of weak bounding cochains defined by

$$
\sum_{k=0}^{\infty} \mathfrak{m}_{k}(b, \ldots, b)=\mathfrak{P}(b) \cdot \operatorname{PD}([L]) .
$$

For $b \in H^{1}(L ; \sqrt{-1} \mathbb{R}) \subset H^{*}\left(L ; \Lambda_{0}\right)$ satisfying the Maurer-Cartan equation, the potential function is naively given by

$$
\begin{aligned}
& \mathfrak{P}(b)=\sum_{\substack{\beta \in \pi_{2}(X, L), \mu_{L}(\beta)=2}} n_{\beta}(L) z_{\beta}(b), \\
& z_{\beta}(b)=\operatorname{hol}_{b}(\partial \beta) T^{\int_{\beta} \omega},
\end{aligned}
$$

where $\mu_{L}$ is the Maslov index, $\operatorname{hol}_{b}(\partial \beta)$ is the holonomy of $b$ regarded as a flat $U(1)$-connection on $L$ along the boundary $\partial \beta$, and $n_{\beta}(L)$ is the "number" of pseudo-holomorphic disks in the class $\beta$ bounded by $L$ defined by

$$
\mathrm{ev}_{*}\left[\overline{\mathcal{M}}_{1}(X, L ; \beta)\right]=n(\beta)[L] .
$$

Cho and Oh [CO06] and Fukaya, Oh, Ohta and Ono [FOOO10] computed the potential functions for Lagrangian torus orbits in toric manifolds. Combining this with Theorem 4.2 , one can compute the potential function of Lagrangian torus fibers $L(\boldsymbol{u})=L_{\Gamma}(\boldsymbol{u})$ of the completely integrable system $\Psi_{\Gamma}$ on $\operatorname{Gr}(2, n)$. Let

$$
\ell_{i}(\boldsymbol{u})=\left\langle\boldsymbol{v}_{i}, \boldsymbol{u}\right\rangle-\tau_{i} \geq 0
$$

be the defining inequalities of $\Delta_{\Gamma}$ given in (3.35), (3.36), (3.37);

$$
\Delta_{\Gamma}=\left\{\boldsymbol{u} \in \mathbb{R}^{2 n-4} \mid \ell_{i}(\boldsymbol{u}) \geq 0, i=1, \ldots, m\right\} .
$$

Recall that a holomorphic disk in the toric variety $X_{\Gamma}$ of Maslov index two bounded by a Lagrangian torus orbit intersect transversally a unique toric divisor at one point. Let $\beta_{i} \in \pi_{2}(\operatorname{Gr}(2, n), L(\boldsymbol{u}))$ denote the class of a pseudo-holomorphic disk which is deformed into that in $X_{\Gamma}$ intersecting a toric divisor corresponding to the codimension one face $\left\{\ell_{i}(\boldsymbol{u})=0\right\}$ of $\Delta_{\Gamma}$. 
Theorem 5.1 (N14, Theorem 8.1]). For any $\boldsymbol{u} \in \operatorname{Int} \Delta_{\Gamma}$, one has an inclusion $H^{1}\left(L(\boldsymbol{u}) ; \Lambda_{0}\right) \subset \mathcal{M}(L(\boldsymbol{u}))$, and the potential function of $L(\boldsymbol{u})$ is given by

$$
\begin{aligned}
\mathfrak{P}_{\Gamma}(L(\boldsymbol{u}), \boldsymbol{x}) & =\sum_{i=1}^{m} z_{\beta_{j}}(\boldsymbol{u}, \boldsymbol{x}), \\
z_{\beta_{i}}(\boldsymbol{u}, \boldsymbol{x}) & =e^{\left.\boldsymbol{v}_{i}, x\right\rangle} T^{\ell_{i}(\boldsymbol{u})}
\end{aligned}
$$

for $\boldsymbol{x}=\left(x_{i j}\right)_{\epsilon(i, j) \in \operatorname{Prn} \Gamma} \in H^{1}\left(L(\boldsymbol{u}) ; \Lambda_{0}\right) \cong \Lambda_{0}^{2 n-4}$.

By setting $y_{i j}=T^{u_{i j}} e^{x_{i j}}$ for $\epsilon(i, j) \in \operatorname{Prn} \Gamma$ and $q=T^{\lambda}$, we have a Laurent polynomial

$$
W_{\Gamma}:\left(\mathbb{G}_{m}\right)^{2 n-4} \longrightarrow \mathbb{A}^{1}
$$

in $\boldsymbol{y}=\left(y_{i j}\right)_{\epsilon(i, j) \in \operatorname{Prn} \Gamma}$ defined by

$$
\mathfrak{P}_{\Gamma}(L(\boldsymbol{u}), \boldsymbol{x})=W_{\Gamma}(\boldsymbol{y}, q) .
$$

For $1 \leq i<j \leq n$, define a new variable $y(i, j)$ corresponding to $u(i, j)$ by

$$
y(i, j)= \begin{cases}y_{i, i+1}^{1 / 2}, & j=i+1<n+1, \\ q /\left(\prod_{k=1}^{n-1} y_{k, k+1}\right)^{1 / 2}, & (i, j)=(1, n), \\ y_{i j} /\left(\prod_{k=i}^{j-1} y_{k, k+1}\right)^{1 / 2}, & |i-j| \geq 2 .\end{cases}
$$

Then $W_{\Gamma}$ is given by

$$
W_{\Gamma}=\sum\left(\frac{y(i, j) y(j, k)}{y(i, k)}+\frac{y(i, j) y(i, k)}{y(j, k)}+\frac{y(i, k) y(j, k)}{y(i, j)}\right),
$$

where the sum is taken over all triangles in the triangulation.

Example 5.2. Recall that the polytope $\Delta_{\Gamma_{\text {cat }}}$ corresponding to the caterpillar $\Gamma_{\text {cat }}$ is given by (3.39). Then the potential function is given by

$$
\begin{aligned}
W_{\Gamma_{\text {cat }}} & =\frac{y_{1,3}}{y_{1,2}}+\frac{y_{1,4}}{y_{1,3}}+\cdots+\frac{y_{1, n-1}}{y_{1, n-2}}+\frac{q}{y_{1, n-1}} \\
+ & \frac{y_{1,2} y_{1,3}}{\prod_{k=1}^{2} y_{k, k+1}}+\frac{y_{1,3} y_{1,4}}{\prod_{k=1}^{3} y_{k, k+1}}+\cdots+\frac{y_{1, n-2} y_{1, n-1}}{\prod_{k=1}^{n-2} y_{k, k+1}}+\frac{q y_{1, n-1}}{\prod_{k=1}^{n-1} y_{k, k+1}} \\
& +\frac{y_{1,2} y_{2,3}}{y_{1,3}}+\frac{y_{1,3} y_{3,4}}{y_{1,4}}+\cdots+\frac{y_{1, n-2} y_{n-2, n-1}}{y_{1, n-1}}+\frac{y_{1, n-1} y_{n-1, n}}{q} .
\end{aligned}
$$

Proposition 5.3. Let $\Gamma$ and $\Gamma^{\prime}$ be two triangulation related by a Whitehead move in a quadrilateral with vertices $a, b, c, d(1 \leq a<b<c<d \leq n)$. Then the corresponding potential functions $W_{\Gamma}, W_{\Gamma^{\prime}}$ are related by the geometric lift

$$
y_{a c} y_{b d}=\frac{y_{a b} y_{b c} y_{c d} y_{a d}}{y_{a b} y_{c d}+y_{a d} y_{b c} / \prod_{i=b}^{c-1} y_{i, i+1}}
$$

of the piecewise linear transformation (3.41] in the sense of [BZ01]. 
Proof. Setting $y_{1}=y(a, b), y_{2}=y(b, c), y_{3}=y(c, d), y_{4}=y(a, d)$ and $y=y(a, c), y^{\prime}=y(b, d)$, the potential functions corresponding to $\Gamma$ and $\Gamma^{\prime}$ can be written as

$$
\begin{aligned}
W_{\Gamma} & =\frac{y_{1} y}{y_{2}}+\frac{y_{2} y}{y_{1}}+\frac{y_{1} y_{2}}{y}+\frac{y_{3} y}{y_{4}}+\frac{y_{4} y}{y_{3}}+\frac{y_{3} y_{4}}{y}+F(\boldsymbol{y}) \\
& =\frac{\left(y_{1} y_{3}+y_{2} y_{4}\right)\left(y_{1} y_{4}+y_{2} y_{3}\right)}{y_{1} y_{2} y_{3} y_{4}} \cdot y+\frac{y_{1} y_{2}+y_{3} y_{4}}{y}+F(\boldsymbol{y}), \\
W_{\Gamma^{\prime}} & =\frac{\left(y_{1} y_{3}+y_{2} y_{4}\right)\left(y_{1} y_{2}+y_{3} y_{4}\right)}{y_{1} y_{2} y_{3} y_{4}} \cdot y^{\prime}+\frac{y_{1} y_{4}+y_{2} y_{3}}{y^{\prime}}+F(\boldsymbol{y}),
\end{aligned}
$$

for a Laurent polynomial $F(\boldsymbol{y})$ independent of $y, y^{\prime}$. 3.50) implies that the coordinate change (5.17) is given by

$$
y^{\prime}=\frac{1}{y} \cdot \frac{y_{1} y_{2} y_{3} y_{4}}{y_{1} y_{3}+y_{2} y_{4}},
$$

which transforms $W_{\gamma}$ into $W_{\Gamma^{\prime}}$.

\section{Cluster algebras}

The homogeneous coordinate ring $\mathbb{C}[\operatorname{Gr}(2, n)]$ is generated by $\left\{p_{i j}\right\}_{1 \leq i<j \leq n}$ with Plücker relations (1.6) ${ }^{2}$ It is a prototypical example of a cluster algebra defined by a quiver, also known as a skew-symmetric cluster algebra of geometric type. The notion of cluster algebras is introduced in [FZ02]. The cluster algebra structure on the homogeneous coordinate ring of $\operatorname{Gr}(2, n)$ is established in [FZ03], which is generalized to $\operatorname{Gr}(k, n)$ in [Sco06. It is also the cluster algebra associated with a disk with $n$ marked points on the boundary, which is a special case of a cluster algebra associated with a bordered surface with marked points [FG06, GSV05, FST08].

A quiver $Q=\left(Q_{0}, Q_{1}, s, t\right)$ consists of a set $Q_{0}$ of vertices, a set $Q_{1}$ of arrows, and two maps $s, t: Q_{1} \rightarrow Q_{0}$ sending an arrow to its source and target respectively.

A disk with $n$ marked points is homeomorphic to the reference polygon $P$ with $n$ sides. With each triangulation $\Gamma$ of the reference polygon $P$, we associate a quiver $Q_{\Gamma}$ as shown in Figure 6.1. The arrows in $Q_{\Gamma}$ are oriented in such a way that each cycle contained in a triangle of the triangulation is oriented clockwise. Boxed vertices on the boundary corresponding to edges of $P$ are frozen, and circled vertices in the interior corresponding to diagonals in $\Gamma$ are mutable. For a mutable vertex $v$ in the quiver $Q_{\Gamma}$, the mutated quiver $\mu_{v}\left(Q_{\Gamma}\right)$ is constructed in three steps:

\footnotetext{
${ }^{2}$ The Grassmannian $\operatorname{Gr}(2, n)=\operatorname{Gr}\left(2, \mathbb{C}^{n}\right)$ in this section is canonically identified with its dual Grassmannian $\operatorname{Gr}\left(n-2,\left(\mathbb{C}^{n}\right)^{*}\right)$, which appears in the B-model side. Hence the indices of the Plücker coordinates are labels of vertices of $P$.
} 


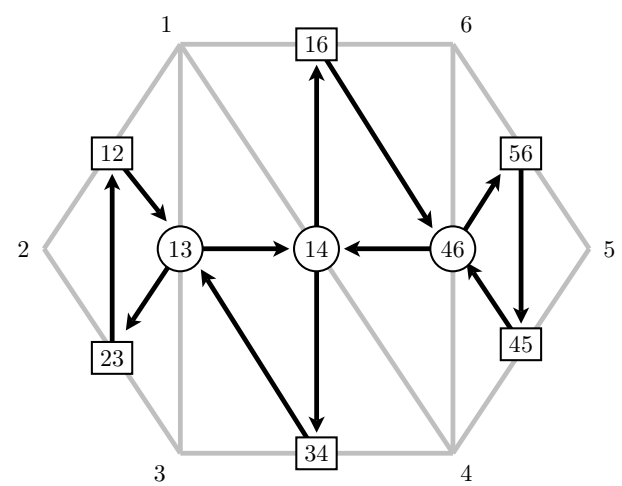

Figure 6.1: A quiver

1. For each path $u \rightarrow v \rightarrow w$ of length two passing through $v$, add a new arrow $u \rightarrow w$.

2. Reverse all arrows with source or target $v$.

3. Annihilate pairs of arrows $a, b$ with $s(a)=t(b)$ and $t(a)=s(b)$, in such a way that no oriented 2 -cycle (i.e., a path of length two with the same source and the target) remains.

One can easily see that Whitehead moves of triangulations correspond to mutations of associated quivers.

We name mutable vertices as $v_{1}, \ldots, v_{n-3}$, and frozen vertices as $v_{n-2}, \ldots, v_{2 n-3}$. With each vertex $v_{i}$, we associate a variable $\mathrm{x}_{i}$, which is called a cluster variable if $i=1, \ldots, n-3$ and a frozen (or coefficient) variable if $i=n-2, \ldots, 2 n-3$. The sequence $\mathbf{x}=\left(\mathrm{x}_{1}, \ldots, \mathrm{x}_{n-3}\right)$ is called a cluster. The pair $\left(\mathbf{x}, Q_{\Gamma}\right)$ of a cluster and a quiver is called a labeled seed. Under the mutation $\mu_{v}$ of the quiver $Q_{\Gamma}$ at the vertex $v$, the labeled seed is transformed as $\left(\mathbf{x}, Q_{\Gamma}\right) \mapsto\left(\mathbf{x}^{\prime}, \mu_{v}\left(Q_{\Gamma}\right)\right)$, where $\mathbf{x}_{w}^{\prime}=\mathrm{x}_{w}$ for $v \neq w$ and

$$
\mathrm{x}_{v}^{\prime} \mathrm{x}_{v}=\prod_{s(a)=v} \mathrm{x}_{t(a)}+\prod_{t(a)=v} \mathrm{x}_{s(a)} .
$$

The cluster algebra is the $\mathbb{Z}\left[\mathrm{x}_{n-2}, \ldots, \mathrm{x}_{2 n-3}\right]$-subalgebra of the ambient field $\mathbb{Q}\left(\mathrm{x}_{1}, \ldots, \mathrm{x}_{2 n-3}\right)$ generated by cluster variables in all the seeds obtained from the initial seed $\left(\mathbf{x}, Q_{\Gamma}\right)$ by any sequence of mutations. One can easily see that the cluster transformation (6.1) for the Whitehead move interchanging the diagonals $d_{i k}$ and $d_{j l}$ gives exactly the Plücker relation (1.6). It follows that the cluster algebra in this case is the homogeneous coordinate ring of $\operatorname{Gr}(2, n)$. 


\section{Landau-Ginzburg mirrors}

The mirror of $\operatorname{Gr}(2, n)$ is identified with the Landau-Ginzburg model

$$
\left(\check{X}, W=\sum_{i=1}^{n} \frac{p_{i, i+2}}{p_{i, i+1}} q^{\delta_{i, n-1}}: \check{X} \rightarrow \mathbb{A}^{1}\right)
$$

by Marsh and Rietsch [MR], where $\delta_{i, n-1}$ is the Kronecker delta, and $\check{X}:=$ $\operatorname{Gr}(2, n) \backslash D$ is the complement of an anti-canonical divisor

$$
D=\left\{p_{12}=0\right\} \cup\left\{p_{23}=0\right\} \cup \cdots \cup\left\{p_{n-1, n}=0\right\} \cup\left\{p_{1, n}=0\right\} .
$$

Here $\left(p_{i j}\right)_{1 \leq i<j \leq n}$ is the Plücker coordinate on $\operatorname{Gr}(2, n)$ and $q=T^{\lambda}$ is an element of the quotient field $\Lambda$ of the Novikov ring $\Lambda_{0}$. The arrow $W$ in (7.1) is a morphism of algebraic varieties over $\Lambda$. The Landau-Gizburg model (7.1) is a special case of [Rie08, where Landau-Ginzburg mirrors of general flag varieties are introduced.

An open subspace of this Landau-Ginzburg model is given earlier in [EHX97, BCFKvS98, BCFKvS00]: Consider a quiver $Q=\left(Q_{0}, Q_{1}, s, t\right)$ of the form

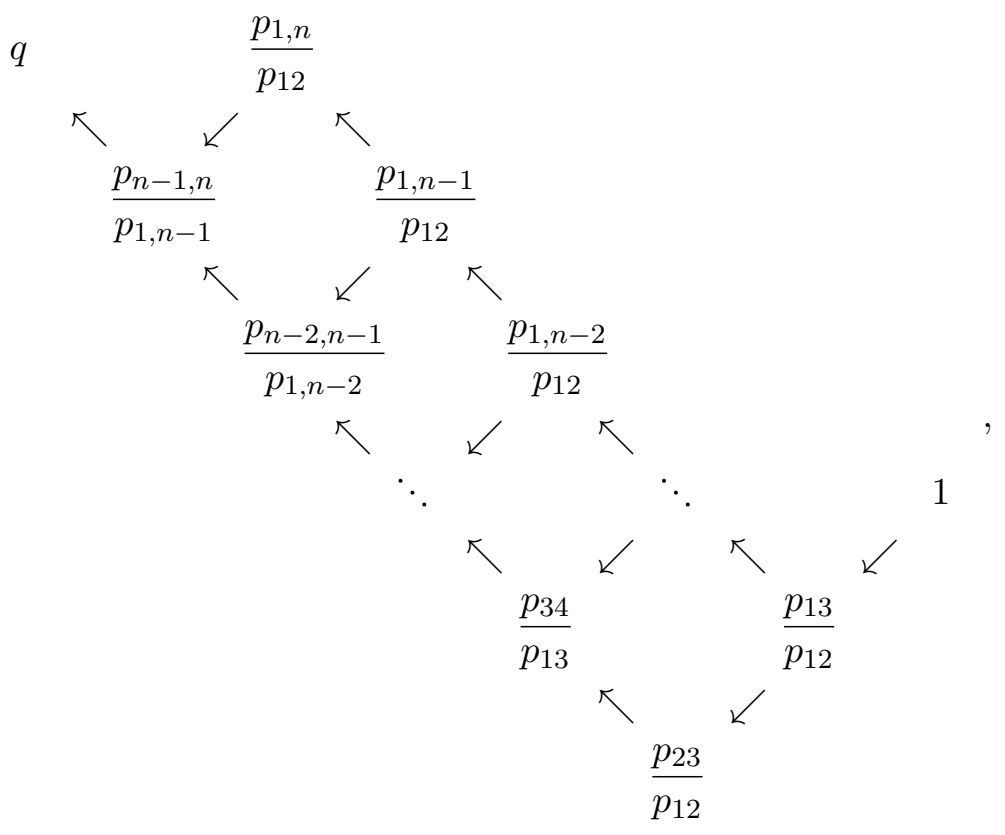

where vertices are Laurent monomials in the Plücker coordinate. It is shown in [MR, Proposition 5.9] that the restriction of the Landau-Ginzburg potential (7.1) to the torus in $\operatorname{Gr}(2, n)$ defined by $p_{i, i+1} \neq 0$ for $i=1, \ldots, n-1$ and $p_{1, i} \neq 0$ for $i=1, \ldots, n-1$ is given by

$$
W=\sum_{a \in Q_{1}} \frac{t(a)}{s(a)} .
$$


For each triangulation $\Gamma$ of the reference polygon, define an open embedding $\iota_{\Gamma}: U_{\Gamma}:=\left(\mathbb{G}_{m}\right)^{\operatorname{Prn} \Gamma} \hookrightarrow \check{X}$ by

$$
y_{i j}= \begin{cases}q \frac{p_{1 n}}{p_{i n}}, & i=2,3, \ldots, n-1 \text { and } j=n, \\ \frac{p_{j, j+1}}{p_{i j}}, & \text { otherwise. }\end{cases}
$$

Remark 7.1. Applying (7.5) formally to the case $(i, j)=(1, n)$, we obtain $y_{1 n}=q$, which is consistent with the fact that $\psi_{1 n}=\lambda$ is constant.

Theorem 7.2. $\quad$ 1. For each triangulation $\Gamma$ of the reference polygon, the potential function $W_{\Gamma}$ is the restriction of the Marsh-Rietsch superpotential (7.1);

$$
W_{\Gamma}=\iota_{\Gamma}^{*} W
$$

2. Let $\Gamma$ and $\Gamma^{\prime}$ be two triangulation related by a Whitehead move in a quadrilateral with vertices $a, b, c, d(1 \leq a<b<c<d \leq n)$. Then the transformation (5.17) is equivalent to the Plücker relation

$$
p_{a c} p_{b d}=p_{a b} p_{c d}+p_{a d} p_{b c} .
$$

under the coordinate change 7.5 .

Proof. In the case of caterpillar $\Gamma_{\text {cat }}$, it is straightforward to see from (5.16) (or (3.39) ) and (7.3) that the Landau-Ginzburg potential (7.4) is identified with the potential function (5.10) under the coordinate change (7.5). One can also easily check that the coordinate change (5.17), which can be written as

$$
\frac{1}{y_{a c} y_{b d}}=\frac{1}{y_{a b} y_{c d} \prod_{i=b}^{c-1} y_{i, i+1}}+\frac{1}{y_{a d} y_{b c}}
$$

is equivalent to the Plücker relation (7.7). This implies that $U_{\Gamma}$ and $U_{\Gamma^{\prime}}$ are glued together in $\check{X} \subset \operatorname{Gr}(2, n)$. Since any triangulation is related to the caterpillar by a sequence of Whitehead moves, Proposition 5.3 prove the first statement for any $\Gamma$.

Remark 7.3. The union $\bigcup_{\Gamma} U_{\Gamma}$ does not cover the whole $\check{X}$ in general. In the case of $\operatorname{Gr}(2,4)$, the complement of the open subset $\bigcup_{\Gamma} U_{\Gamma}$ is given by

$$
\check{X} \backslash \bigcup_{\Gamma} U_{\Gamma}=\left\{\left[p_{i j}\right] \in \check{X} \mid p_{13}=p_{24}=0\right\} \cong\left(\mathbb{G}_{m}\right)^{2} .
$$

In this case, the superpotential $W$ has two critical points with zero critical value, which are contained in this complement. We expect that singular Lagrangian fibers of the Lagrangian torus fibration interpolating $\Psi_{\Gamma}$ for different $\Gamma$ correspond to points in this complement under homological mirror symmetry just as in [CPU16, Section 8]. See also [NU16, CKO, CK]. 


\section{Wall-crossing formula}

Let $\Phi: X \rightarrow B$ be a Lagrangian torus fibration on a symplectic manifold $(X, \omega)$ possibly with singular fibers.

Definition 8.1. A Lagrangian fiber $L(\boldsymbol{u})=\Phi^{-1}(\boldsymbol{u})$ is said to be potentially obstructed if it bounds a pseudo-holomorphic disk of Maslov index zero. The set of $\boldsymbol{u} \in B$ with potentially obstructed fiber $L(\boldsymbol{u})$ is called a wall.

We assume that any wall has codimension one, and any Lagrangian fiber $L(\boldsymbol{u})$ satisfies the following conditions([Aur07, Assumptions 3.2, 3.8]):

- there are no non-constant holomorphic sphere $v: \mathbb{P}^{1} \rightarrow X$ with $c_{1}(T X)$. $[v] \leq 0$

- holomorphic disks of Maslov index two in $(X, L(\boldsymbol{u}))$ are regular;

- all simple (non multiply covered) non-constant holomorphic disks in $(X, L(\boldsymbol{u}))$ of Maslov index zero are regular, and the associated evaluation maps at boundary marked points are transverse to each other and to the evaluation maps at boundary marked points of holomorphic disks of Maslov index 2.

Let $U_{0}, U_{1} \subset B$ be two chambers separated by a wall on which each fiber bounds a unique non-constant pseudo-holomorphic disk of Maslov index zero. Let $\alpha \in \pi_{2}(X, L(\boldsymbol{u}))$ be the class of such a disk.

Proposition 8.2 (Auroux Aur07, Proposition 3.9]). For $\boldsymbol{u}_{0} \in U_{0}$ and $\boldsymbol{u}_{1} \in U_{1}$, the functions $z_{\beta}$ given in (5.6) defined for $L\left(\boldsymbol{u}_{0}\right)$ and $L\left(\boldsymbol{u}_{1}\right)$ are related by

$$
z_{\beta} \longmapsto z_{\beta} h\left(z_{\alpha}\right)^{[\partial \alpha] \cdot[\partial \beta]}
$$

for a function $h\left(z_{\alpha}\right)=1+O\left(z_{\alpha}\right)$.

This wall-crossing formula also follows from the isomorphism of filtered $A_{\infty}$-algebras associated with the change of almost complex structures given in [FOOO09].

Now we recall an example of the wall-crossing formula given by Auroux in [Aur07, Section 5] and [Aur09, Section 3.1].

Example 8.3. Consider the $\mathbb{C}^{\times}$-action on

$$
Y=\left\{\left(x_{1}, x_{2}, x_{3}\right) \in \mathbb{C}^{3} \mid x_{1} x_{2}=1+x_{3}\right\} \cong \mathbb{C}^{2}=\mathbb{C}_{x_{1}} \times \mathbb{C}_{x_{2}}
$$

defined by

$$
\tau \cdot\left(x_{1}, x_{2}, x_{3}\right)=\left(\tau x_{1}, \tau^{-1} x_{2}, x_{3}\right), \quad \tau \in \mathbb{C}^{\times} .
$$


Then the projection $f: Y \rightarrow \mathbb{C},\left(x_{1}, x_{2}, x_{3}\right) \mapsto x_{3}$ gives the GIT quotient with respect to the $\mathbb{C}^{\times}$-action. Equip $Y$ with an $S^{1}\left(\subset \mathbb{C}^{\times}\right)$-invariant symplectic form, and let $\mu_{S^{1}}: Y \rightarrow \mathbb{R}$ be the moment map of the $S^{1}$-action. Define a Lagrangian torus fibration on $Y$ by

$$
\Phi: Y \rightarrow \mathbb{R}_{\geq 0} \times \mathbb{R}, \quad x=\left(x_{1}, x_{2}, x_{3}\right) \mapsto\left(\left|x_{3}\right|, \mu_{S^{1}}(x)\right),
$$

and write its fibers as

$$
T_{R, r}=\Phi^{-1}(R, r)=\left\{x \in Y|| x_{3} \mid=R, \mu_{S^{1}}(x)=r,\right\} .
$$

This fibration has a unique singular fiber $T_{1,0}$ over $(1,0)$, which is a twotorus with one circle pinched. For $r \neq 0$, each fiber $T_{1, r}$ over the line $R=1$ intersects a coordinate axis of $Y$ viewed as $\mathbb{C}_{x_{1}} \times \mathbb{C}_{x_{2}}$ at a circle, and thus it bounds a holomorphic disk of Maslov index zero, which has the form $D^{2} \times\{0\}$ or $\{0\} \times D^{2}$ in $\mathbb{C}_{x_{1}} \times \mathbb{C}_{x_{2}} \cong Y$. Hence the wall of $\Phi$ is given by $R=1$. Let $\alpha \in \pi_{2}\left(Y, T_{1, r}\right)$ denote the class of disks of the form $D^{2} \times\{0\}$. Lagrangian fibers over the chambers $R>1$ and $R<1$ are said to be of Clifford type and of Chekanov type, respectively. A Clifford type fiber $T_{r, R}$ can be deformed into a torus of the form $S^{1}\left(r_{1}\right) \times S^{1}\left(r_{2}\right) \subset \mathbb{C}_{x_{1}} \times \mathbb{C}_{x_{2}}$, which bounds holomorphic disks of Maslov index two of the forms $D^{2}\left(r_{1}\right) \times\{\mathrm{pt}\}$ and $\{\mathrm{pt}\} \times D^{2}\left(r_{2}\right)$. Let $\beta_{1}, \beta_{2} \in \pi_{2}\left(Y, T_{r, R}\right)$ be the classes of these holomorphic disks. On the other hand, a Chekanov type torus $T_{r, R}$ bounds a family of holomorphic disks of Maslov index two, which are sections of $f: Y \rightarrow \mathbb{C}$ over the disk $D^{2}(R)$ enclosed by the image $f\left(T_{r, R}\right)=\left\{x_{3} \in \mathbb{C}|| x_{3} \mid=R\right\}$ of $T_{r, R}$. Let $\beta_{3}$ denote its homotopy class. Then the wall-crossing formula is given by

$$
z_{\beta_{3}}= \begin{cases}z_{\beta_{2}}\left(1+z_{\alpha}\right) & (r>0), \\ z_{\beta_{1}}\left(1+z_{\alpha}^{-1}\right) & (r<0) .\end{cases}
$$

Since $z_{\alpha}=z_{\beta_{1}} / z_{\beta_{2}}$, the transformations 8.6 on $r>0$ and $r<0$ are identical.

Let us go back to the case of the Grassmannian $\operatorname{Gr}(2, n)$. Suppose we have two triangulation $\Gamma, \Gamma^{\prime}$ of the reference polygon related by a Whitehead move in a quadrilateral with vertices $1 \leq a<b<c<d \leq n$, and let $\Gamma^{\prime \prime}$ be the subdivision given by common diagonals in $\Gamma$ and $\Gamma^{\prime}$. Note that $\Psi_{\Gamma}$ and $\Psi_{\Gamma^{\prime}}$ are written as

$$
\Psi_{\Gamma}=\left(\left(\psi_{i j}\right)_{\epsilon(i, j) \in \operatorname{Prn} \Gamma^{\prime \prime}}, \psi_{a c}\right), \quad \Psi_{\Gamma^{\prime}}=\left(\left(\psi_{i j}\right)_{\epsilon(i, j) \in \operatorname{Prn} \Gamma^{\prime \prime}}, \psi_{b d}\right) .
$$

Since $\varphi_{a c}=\psi_{a c}-\frac{1}{2} \sum_{i=a}^{c-1} \psi_{i, i+1}$ and $\varphi_{b d}=\psi_{b d}-\frac{1}{2} \sum_{i=b}^{d-1} \psi_{i, i+1}$ Poisson commute with all other $\psi_{i j}, \epsilon(i, j) \in \operatorname{Prn} \Gamma^{\prime \prime}$, the function

$$
\psi_{t}=(1-t)\left(\varphi_{a c}\right)^{2}-t\left(\varphi_{b d}\right)^{2}
$$


also Poisson commutates with the functions $\psi_{i j}$ for any $t \in[0,1]$ :

$$
\left\{\psi_{t}, \psi_{i j}\right\}=0 .
$$

Hence we obtain the following.

Proposition 8.4. Let $\Gamma, \Gamma^{\prime}$ be two triangulations of $P$ as above. Then

$$
\Psi_{t}=\left(\left(\psi_{i j}\right)_{\epsilon(i, j) \in \operatorname{Prn} \Gamma^{\prime \prime}}, \psi_{t}\right), \quad t \in[0,1]
$$

is a one-parameter family of completely integrable systems on $\operatorname{Gr}(2, n)$ connecting $\Psi_{\Gamma}$ and $\Psi_{\Gamma^{\prime}}$.

Theorem 8.5. Let $\Gamma$ and $\Gamma^{\prime}$ be two triangulations as above. For $t \in(0,1)$, the wall-crossing formula (8.1) for $\Psi_{t}$ is equivalent to the coordinate change (5.17) (and hence, to the Plücker relation (7.7)).

We prove this theorem in the next two sections.

\section{Wall-crossing formula on $\operatorname{Gr}(2,4)$}

In this section we prove Theorem 8.5 in the case of $\operatorname{Gr}(2,4)$.

Let $\Gamma, \Gamma^{\prime}$ be the two triangulations of a quadrilateral $P$ given by diagonals $d_{13}, d_{24}$, respectively. Then the corresponding potential functions

$$
\begin{gathered}
W_{\Gamma}=\frac{y_{13}}{y_{12}}+\frac{y_{13}}{y_{23}}+\frac{y_{12} y_{23}}{y_{13}}+\frac{q y_{13}}{y_{12} y_{23} y_{34}}+\frac{y_{13} y_{34}}{q}+\frac{q}{y_{13}}, \\
W_{\Gamma^{\prime}}=\frac{y_{24}}{y_{23}}+\frac{y_{24}}{y_{23}}+\frac{y_{23} y_{34}}{y_{24}}+\frac{q y_{24}}{y_{12} y_{23} y_{34}}+\frac{y_{12} y_{24}}{q}+\frac{q}{y_{24}}
\end{gathered}
$$

are related by the coordinate change (5.17), which is given by

$$
\frac{1}{y_{13} y_{24}}=\frac{1}{q y_{23}}\left(1+\frac{q}{y_{12} y_{34}}\right)
$$

in this case.

Recall that the image of the moment map $\mu_{\mathbb{T}_{U(4)}}: \operatorname{Gr}(2,4) \cong \mathcal{O}_{\lambda} \rightarrow \mathbb{R}^{4}$ is an octahedron in $\left\{\boldsymbol{r}=\left(r_{1}, \ldots, r_{4}\right) \in \mathbb{R}^{4} \mid \sum_{i=1}^{4} r_{i}=\lambda\right\} \cong \mathbb{R}^{3}$ defined by

$$
0 \leq r_{i} \leq \sum_{j \neq i} r_{j}, \quad i=1, \ldots, 4,
$$

shown in Figure 9.1, and critical values of $\mu_{\mathbb{T}_{U(4)}}$ inside the octahedron $\mu_{\mathbb{T}_{U(4)}}\left(\mathcal{O}_{\lambda}\right)$ form three walls $H_{1} \cup H_{2} \cup H_{3}$, where

$$
\begin{aligned}
& H_{1}=\left\{\boldsymbol{r} \mid r_{1}+r_{2}=r_{3}+r_{4}\right\}, \\
& H_{2}=\left\{\boldsymbol{r} \mid r_{1}+r_{3}=r_{2}+r_{4}\right\}, \\
& H_{3}=\left\{\boldsymbol{r} \mid r_{1}+r_{4}=r_{2}+r_{3}\right\}
\end{aligned}
$$




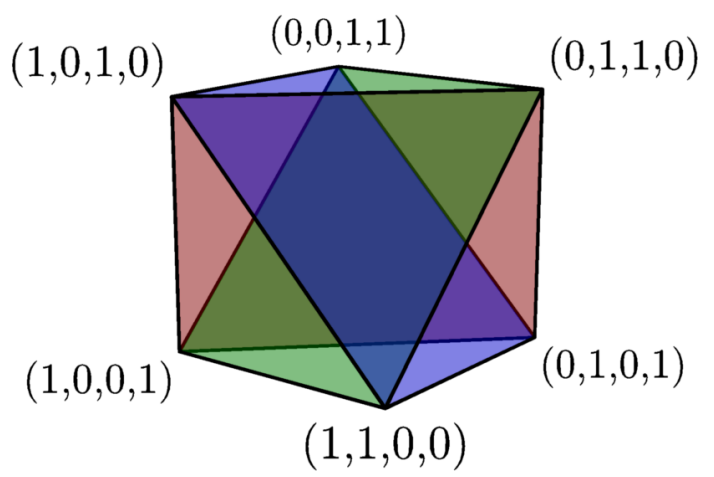

Figure 9.1: The moment polytope of $\mu_{\mathbb{T}_{U(4)}}$ and the hyperplanes $H_{1}$ (red), $H_{2}$ (green), and $H_{3}$ (blue) (rotatable with Acrobat Reader)

(see [HK97, Proposition 4.3] or (9.19) below). For each interior point $\boldsymbol{r}$ in $\mu_{\mathbb{T}_{U(4)}}\left(\mathcal{O}_{\lambda}\right)$, the completely integrable system

$$
\Psi_{t}=\left(\psi_{12}, \psi_{23}, \psi_{34},(1-t)\left(\varphi_{13}\right)^{2}-t\left(\varphi_{24}\right)^{2}\right)
$$

induces the function

$$
\varphi_{t}=(1-t)\left(\varphi_{13}\right)^{2}-t\left(\varphi_{24}\right)^{2}: \mathcal{M}_{\boldsymbol{r}}=\mu_{\mathbb{T}_{U(4)}}^{-1}(\boldsymbol{r}) / \mathbb{T}_{U(4)} \longrightarrow \mathbb{R}
$$

on the polygon space. Let $B_{t}=\Psi_{t}(\operatorname{Gr}(2,4))$ and $I_{t, \boldsymbol{r}}=\varphi_{t}\left(\mathcal{M}_{\boldsymbol{r}}\right)$ denote the ranges of $\Psi_{t}$ and $\varphi_{t}$, respectively. Since $\left(\psi_{12}, \psi_{23}, \psi_{34}\right)$ gives the moment map of the $\mathbb{T}_{U(4)}$-action on $\mathcal{O}_{\lambda}$, we have a natural projection

$$
B_{t} \longrightarrow \mu_{\mathbb{T}_{U(4)}}\left(\mathcal{O}_{\lambda}\right) \subset \mathbb{R}^{3}, \quad\left(u_{1}, u_{2}, u_{3}, u_{4}\right) \longmapsto\left(u_{1}, u_{2}, u_{3}\right) .
$$

Lemma 9.1. For each $t \in(0,1)$ and $\boldsymbol{r} \in \operatorname{Int} \mu_{\mathbb{T}_{U(n)}}\left(\mathcal{O}_{\lambda}\right)$, the map $\varphi_{t}: \mathcal{M}_{\boldsymbol{r}} \rightarrow$ $I_{t, \boldsymbol{r}}$ is an $S^{1}$-bundle over the interior $\operatorname{Int} I_{t, \boldsymbol{r}}$, and the fibers over boundary points of $I_{t, \boldsymbol{r}}$ are single points.

Proof. We first note that the $S^{1}$-fibration $\varphi_{13}: \mathcal{M}_{\boldsymbol{r}} \rightarrow I_{0, \boldsymbol{r}}$ (resp. $\varphi_{24}$ ) is not homeomorphic to the toric moment map on $\mathbb{P}^{1}$ exactly when $\min \varphi_{13}=0$ (resp. $\min \varphi_{24}=0$ ), or equivalently, $r_{1}=r_{2}$ and $r_{3}=r_{4}$ (resp. $r_{1}=r_{4}$ and $r_{2}=r_{3}$ ); in this case, the fiber $\varphi_{13}^{-1}(0)$ consists of "broken lines" $\boldsymbol{\xi}=$ $\left(\xi_{1}, \ldots, \xi_{4}\right)$ satisfying $\xi_{1}+\xi_{2}=\xi_{3}+\xi_{4}=0$, and it is diffeomorphic to a line segment. We consider the map

$$
\varphi=\left(\left(\varphi_{13}\right)^{2},\left(\varphi_{24}\right)^{2}\right): \mathcal{M}_{r} \longrightarrow \mathbb{R}^{2},
$$

and let $\left(v_{1}, v_{2}\right)$ be the standard coordinate on $\mathbb{R}^{2}$. Then the boundary of the image $\varphi\left(\mathcal{M}_{\boldsymbol{r}}\right)$ contains a line segment in a coordinate axis if $\min \varphi_{13}=0$ or $\min \varphi_{24}=0$ (see Figure 9.4 and Figure 9.6). 


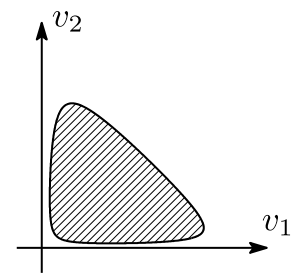

Figure 9.2: $\varphi\left(\mathcal{M}_{\boldsymbol{r}}\right)$ for generic $\boldsymbol{r}$

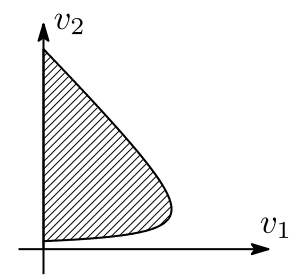

Figure 9.4: $\varphi\left(\mathcal{M}_{\boldsymbol{r}}\right)$ in the case of $\boldsymbol{r} \in \mathrm{H}_{2} \cap \mathrm{H}_{3}$

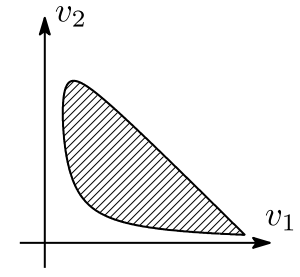

Figure 9.3: $\varphi\left(\mathcal{M}_{\boldsymbol{r}}\right)$ for $\boldsymbol{r} \in H_{1}$

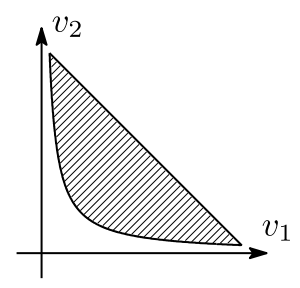

Figure 9.5: $\varphi\left(\mathcal{M}_{\boldsymbol{r}}\right)$ in the case of $\boldsymbol{r} \in H_{1} \cap H_{3}$

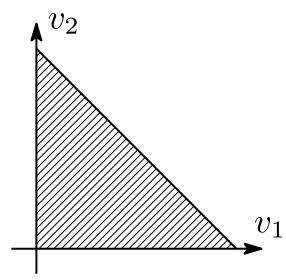

Figure 9.6: $\varphi\left(\mathcal{M}_{\boldsymbol{r}}\right)$ in the case of $\boldsymbol{r} \in H_{1} \cap H_{2} \cap H_{3}$

Claim 9.2. For any $\boldsymbol{r}$ and $t \in(0,1)$, the intersection

$$
\varphi\left(\mathcal{M}_{\boldsymbol{r}}\right) \cap\left\{\left(v_{1}, v_{2}\right) \in \mathbb{R}^{2} \mid(1-t) v_{1}-t v_{2}=c\right\}
$$

is a line segment for $c \in \operatorname{Int} I_{t, \boldsymbol{r}}$, and is a single point in $\partial \varphi\left(\mathcal{M}_{\boldsymbol{r}}\right)$ when $c \in \partial I_{t, \boldsymbol{r}}$.

Assuming Claim 9.2, Lemma 9.1 follows from the fact that $\varphi: \mathcal{M}_{r} \rightarrow$ $\varphi\left(\mathcal{M}_{\boldsymbol{r}}\right)$ is a double cover which branches along the boundary $\partial \varphi\left(\mathcal{M}_{\boldsymbol{r}}\right)$.

Proof of Claim 9.2. Fix $v_{1}>0$ and consider a quadrilateral $\boldsymbol{\xi} \in \mathcal{M}_{\boldsymbol{r}}$ with $\varphi_{13}(\boldsymbol{\xi})=\sqrt{v_{1}}$. Then the angles $\theta_{1}\left(\right.$ resp. $\left.\theta_{4}\right)$ between the side $\xi_{1}$ (resp. $\left.-\xi_{4}\right)$ and the diagonal $\xi_{1}+\xi_{2}$ connecting the first and the third vertices are given by

$$
\cos \theta_{1}=\frac{v_{1}+r_{1}^{2}-r_{2}^{2}}{2 r_{1} \sqrt{v_{1}}}, \quad \cos \theta_{4}=\frac{v_{1}+r_{4}^{2}-r_{3}^{2}}{2 r_{4} \sqrt{v_{1}}}
$$

(see Figure 9.7). Since $\varphi_{24}$ restricted to the level set $\varphi_{13}^{-1}\left(\sqrt{v_{1}}\right)$ takes its maximum and minimum when $\boldsymbol{\xi}$ is contained in a plane, the range of $\left.\left(\varphi_{24}\right)^{2}\right|_{\varphi_{13}^{-1}\left(\sqrt{v_{1}}\right)}$ 


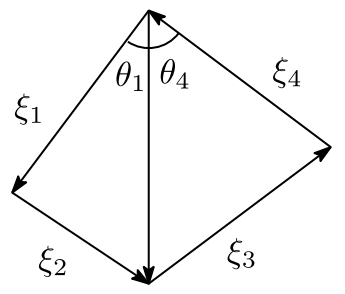

Figure 9.7: A diagonal $\xi_{1}+\xi_{2}$ in a quadrilateral $\boldsymbol{\xi} \in \mathcal{M}_{\boldsymbol{r}}$.

is

$$
\begin{aligned}
r_{1}^{2}+r_{4}^{2}-2 r_{1} r_{4} & \left(\cos \theta_{1} \cos \theta_{4}+\sin \theta_{1} \sin \theta_{4}\right) \\
& \leq v_{2} \leq r_{1}^{2}+r_{4}^{2}-2 r_{1} r_{4}\left(\cos \theta_{1} \cos \theta_{4}-\sin \theta_{1} \sin \theta_{4}\right) .
\end{aligned}
$$

Equality in (9.14) holds if and only if

$$
\cos \theta_{1} \cos \theta_{4} \pm \sin \theta_{1} \sin \theta_{4}=\frac{r_{1}^{2}+r_{4}^{2}-v_{2}}{2 r_{1} r_{4}},
$$

which, combined with 9.13 , gives

$$
\pm \sin \theta_{1} \sin \theta_{4}=\frac{r_{1}^{2}+r_{4}^{2}-v_{2}}{2 r_{1} r_{4}}-\frac{v_{1}+r_{1}^{2}-r_{2}^{2}}{2 r_{1} \sqrt{v_{1}}} \cdot \frac{v_{1}+r_{4}^{2}-r_{3}^{2}}{2 r_{4} \sqrt{v_{1}}} .
$$

By taking the square of the both sides of (9.16) and using

$$
\sin ^{2} \theta_{1} \sin ^{2} \theta_{4}=\left(1-\cos ^{2} \theta_{1}\right)\left(1-\cos ^{2} \theta_{4}\right),
$$

we see that

$$
\begin{aligned}
F\left(v_{1}, v_{2}\right):= & v_{1}^{2} v_{2}+v_{1} v_{2}^{2}-\left(r_{1}^{2}+\right. \\
+\left(r_{1}^{2}-r_{4}^{2}\right)\left(r_{2}^{2}-\right. & \left.\left.r_{3}^{2}\right) r_{1}^{2}+r_{4}^{2}\right) v_{1} v_{2} \\
& +\left(r_{1}^{2}-r_{2}^{2}\right)\left(r_{4}^{2}-r_{2}^{2}+r_{3}^{2}-r_{4}^{2}\right)\left(r_{1}^{2} r_{3}^{2}-r_{2}^{2} r_{4}^{2}\right)
\end{aligned}
$$

gives the defining equation for the boundary of the image $\varphi\left(\mathcal{M}_{\boldsymbol{r}}\right) \subset \mathbb{R}^{2}$. The discriminant of $F\left(v_{1}, v_{2}\right)$ is given by

$$
\begin{aligned}
& r_{1}^{2} r_{2}^{2} r_{3}^{2} r_{4}^{2}\left(r_{1}+r_{2}+r_{3}+r_{4}\right)^{2}\left(-r_{1}+r_{2}+r_{3}+r_{4}\right)^{2} \\
&\left(r_{1}-r_{2}+r_{3}+r_{4}\right)^{2}\left(r_{1}+r_{2}-r_{3}+r_{4}\right)^{2} \\
&\left(r_{1}+r_{2}+r_{3}-r_{4}\right)^{2}\left(r_{1}+r_{2}-r_{3}-r_{4}\right)^{2} \\
& \quad\left(r_{1}-r_{2}+r_{3}-r_{4}\right)^{2}\left(r_{1}-r_{2}-r_{3}+r_{4}\right)^{2},
\end{aligned}
$$

which means that the discriminant locus is $\partial\left(\mu_{\mathbb{T}_{U(4)}}\left(\mathcal{O}_{\lambda}\right)\right) \cup H_{1} \cup H_{2} \cup H_{3}$.

When $\boldsymbol{r}$ does not lie on the discriminant locus, then $\partial\left(\varphi\left(\mathcal{M}_{\boldsymbol{r}}\right)\right)$ is the positive real part of a smooth plane cubic curve. A smooth real plane cubic curve has either 
- one non-compact connected component, or

- one non-compact connected component and one compact connected component.

Only the latter can happen in our case, and the boundary $\partial\left(\varphi\left(\mathcal{M}_{\boldsymbol{r}}\right)\right)$ of $\varphi\left(\mathcal{M}_{\boldsymbol{r}}\right)$ is the compact connected component of the cubic curve. When $\boldsymbol{r}$ lies on exactly one wall, say $H_{1}$, then the resulting cubic curve has one node, and $\varphi\left(\mathcal{M}_{\boldsymbol{r}}\right)$ is the closure of the compact connected component of the complement of the nodal cubic curve (see Figure 9.3). In either case, $\varphi\left(\mathcal{M}_{\boldsymbol{r}}\right)$ is strictly convex.

Next we consider the case where $\boldsymbol{r}$ lies on exactly two walls. If $\boldsymbol{r}$ lies in $H_{2}$ and $H_{3}$, one has $r_{1}=r_{2} \neq r_{3}=r_{4}$, so that

$$
F\left(v_{1}, v_{2}\right)=v_{1}\left(\left(r_{1}^{2}-r_{3}^{2}\right)^{2}+v_{1} v_{2}-2\left(r_{1}^{2}+r_{3}^{2}\right) v_{2}+v_{2}^{2}\right)
$$

and $\varphi\left(\mathcal{M}_{\boldsymbol{r}}\right)$ is bounded by a hyperbola and the $v_{2}$-axis. Similarly, if $\boldsymbol{r}$ lies in $H_{1} \cap H_{2}$, the image $\varphi\left(\mathcal{M}_{\boldsymbol{r}}\right)$ is bounded by a hyperbola and the $v_{1}$-axis. When $\boldsymbol{r}$ lies in $H_{1} \cap H_{3}$, one has $r_{1}=r_{3} \neq r_{2}=r_{4}$, and thus

$$
F\left(v_{1}, v_{2}\right)=\left(v_{1}+v_{2}-2\left(r_{1}^{2}+r_{2}^{2}\right)\right)\left(v_{1} v_{2}-\left(r_{1}^{2}-r_{2}^{2}\right)^{2}\right),
$$

which means that $\varphi\left(\mathcal{M}_{\boldsymbol{r}}\right)$ is bounded by a hyperbola and a line as in Figure 9.5 .

When $\boldsymbol{r}$ lies on all the three walls $H_{1}, H_{2}$ and $H_{3}$, then one has $r_{1}=$ $r_{2}=r_{3}=r_{4}$, so that

$$
F\left(v_{1}, v_{2}\right)=v_{1} v_{2}\left(v_{1}+v_{2}-4 r_{1}^{2}\right)
$$

and $\varphi\left(\mathcal{M}_{\boldsymbol{r}}\right)$ is a triangle.

In all these cases, the line $\left\{\left(v_{1}, v_{2}\right) \mid(1-t) v_{1}-t v_{2}=c\right\}$ intersects $\varphi\left(\mathcal{M}_{\boldsymbol{r}}\right)$ in a line segment or a single boundary point.

For a fixed interior point $\boldsymbol{r}$ in $\mu_{\mathbb{T}_{U(4)}}\left(\mathcal{O}_{\lambda}\right)$, we identify the symplectic reduction $\mathcal{M}_{\boldsymbol{r}}=\mu_{\mathbb{T}_{U(4)}^{-1}}^{-1}(2 \boldsymbol{r}) / \mathbb{T}_{U(4)}$ with the GIT quotient $\operatorname{Gr}(2,4) / / \mathbb{T}_{U(4)}^{\mathbb{C}}$. Recall that $\operatorname{Gr}(2,4) / / \mathbb{T}_{U(4)}^{\mathbb{C}}$ is embedded into $\mathbb{P}^{2}$ by

$$
\operatorname{Gr}(2,4) / / \mathbb{T}_{U(4)}^{\mathbb{C}} \hookrightarrow \mathbb{P}^{2}, \quad\left[p_{i j}\right] \mapsto\left[p_{12} p_{34}: p_{13} p_{24}: p_{14} p_{23}\right],
$$

and its image is given by

$$
\left\{\left[\zeta_{1}: \zeta_{2}: \zeta_{3}\right] \in \mathbb{P}^{2} \mid \zeta_{2}=\zeta_{1}+\zeta_{3}\right\} \cong \mathbb{P}^{1} .
$$

Let $D^{\prime}$ be a divisor in $\operatorname{Gr}(2,4)$ defined by

$$
D^{\prime}=\left\{p_{12}=0\right\} \cup\left\{p_{23}=0\right\} \cup\left\{p_{34}=0\right\},
$$


which is contained in an anti-canonical divisor

$$
D=\left\{p_{12}=0\right\} \cup\left\{p_{23}=0\right\} \cup\left\{p_{34}=0\right\} \cup\left\{p_{14}=0\right\} .
$$

Recall that the defining equation for $Y \subset \mathbb{C}^{3}$ given in $(8.2)$ is

$$
x_{1} x_{2}=1+x_{3} .
$$

Then $\operatorname{Gr}(2,4) \backslash D^{\prime}$ is identified with $Y \times\left(\mathbb{C}^{\times}\right)^{2}$ by

$$
\left[p_{12}: p_{13}: p_{14}: p_{23}: p_{24}: p_{34}\right]=\left[1: x_{1}: s_{1}^{-1} s_{2} x_{3}: s_{1}: s_{2} x_{2}: s_{2}\right] \text {, }
$$

or equivalently,

$$
\left(x_{1}, x_{2}, x_{3}, s_{1}, s_{2}\right)=\left(\frac{p_{13}}{p_{12}}, \frac{p_{24}}{p_{34}}, \frac{p_{14} p_{23}}{p_{12} p_{34}}, \frac{p_{23}}{p_{12}}, \frac{p_{34}}{p_{12}}\right) .
$$

We equip $Y \times\left(\mathbb{C}^{\times}\right)^{2}$ with the symplectic structure induced from that on $\operatorname{Gr}(2,4)$. The $\mathbb{T}_{U(4)}$-action

$$
\tau \cdot\left[p_{i j}\right]_{1 \leq i<j \leq 4}=\left[\tau_{i} \tau_{j} p_{i j}\right], \quad \tau=\operatorname{diag}\left(\tau_{1}, \ldots, \tau_{4}\right) \in \mathbb{T}_{U(4)}
$$

on $\operatorname{Gr}(2,4)$ induces a $\mathbb{T}_{U(4)}$-action on $Y \times\left(\mathbb{C}^{\times}\right)^{2}$ given by

$$
\tau \cdot\left(x_{1}, x_{2}, x_{3}, s_{1}, s_{2}\right)=\left(\frac{\tau_{3}}{\tau_{2}} x_{1}, \frac{\tau_{2}}{\tau_{3}} x_{2}, x_{3}, \frac{\tau_{3}}{\tau_{1}} s_{1}, \frac{\tau_{3} \tau_{4}}{\tau_{1} \tau_{2}} s_{2}\right) .
$$

Then the projection $Y \times\left(\mathbb{C}^{\times}\right)^{2} \rightarrow \mathbb{C}_{x_{3}}$ to the $x_{3}$-plane is identified with the restriction to $\operatorname{Gr}(2,4) \backslash D^{\prime}$ of the GIT quotient of $\operatorname{Gr}(2,4)$ by the $\mathbb{T}_{U(4)}$-action:

$$
\begin{array}{cccc}
Y \times\left(\mathbb{C}^{\times}\right)^{2} & \hookrightarrow & \operatorname{Gr}(2,4)^{\mathrm{ss}} \\
\downarrow & & \downarrow \\
\mathbb{C} & \hookrightarrow & \operatorname{Gr}(2,4) / / / \mathbb{T}_{U(4)}^{\mathbb{C}},
\end{array}
$$

where the inclusion $\mathbb{C} \hookrightarrow \operatorname{Gr}(2,4) / / \mathbb{T}_{U(4)}^{\mathbb{C}}$ is given by $x_{3}=\zeta_{3} / \zeta_{1}$.

Lemma 9.3. For each $\boldsymbol{r}$, the bending Hamiltonians $\varphi_{13}: \mathcal{M}_{\boldsymbol{r}} \rightarrow \mathbb{R}$ and $\varphi_{24}: \mathcal{M}_{\boldsymbol{r}} \rightarrow \mathbb{R}$ take their maximums at the points $\zeta_{1}=0$ and $\zeta_{3}=0$ respectively under the inclusion (9.23).

Proof. Assume $r_{1}+r_{2} \leq r_{3}+r_{4}$ so that $\max \varphi_{13}=r_{1}+r_{2}$. Take a point $\left[z_{i}, w_{i}\right]_{i} \in \operatorname{Gr}(2,4)=\operatorname{Mat}_{4 \times 2}(\mathbb{C}) / /{ }_{\lambda} U(2)$ such that $\varphi_{13}$ attains its maximum at $\boldsymbol{\xi}=\left(\nu\left(z_{i}, w_{i}\right)\right)_{i=1, \ldots, 4} \in \mathcal{M}_{\boldsymbol{r}}$, where $\nu$ is the Hopf fibration given in (3.22). Since the side vectors $\xi_{1}=\nu\left(z_{1}, w_{1}\right)$ and $\xi_{2}=\nu\left(z_{2}, w_{2}\right)$ of $\boldsymbol{\xi}$ have the same direction, $\left(z_{1}, w_{1}\right)$ and $\left(z_{2}, w_{2}\right)$ are proportional, which implies that

$$
p_{12}=\operatorname{det}\left(\begin{array}{ll}
z_{1} & w_{1} \\
z_{2} & w_{2}
\end{array}\right)=0 .
$$

Hence we obtain $\zeta_{1}=p_{12} p_{34}=0$. In the case where $r_{1}+r_{2} \geq r_{3}+r_{4}$, we have $p_{34}=0$ at $\left[z_{i}, w_{i}\right]_{i} \in \operatorname{Gr}(2,4)$ such that $\max \varphi_{13}$ is attained at $\boldsymbol{\xi}=\left(\nu\left(z_{i}, w_{i}\right)\right)_{i} \in \mathcal{M}_{\boldsymbol{r}}$, which leads to the same conclusion. The proof for $\varphi_{24}$ is similar. 
Remark 9.4. The points $\zeta_{1}=0$ and $\zeta_{3}=0$ corresponds to $x_{3}=\infty$ and $x_{3}=0$ respectively. The point $\zeta_{2}=p_{13} p_{24}=0$ corresponding to $x_{3}=-1$ is given by quadrangles satisfying $\xi_{1} \| \xi_{3}$ or $\xi_{2} \| \xi_{4}$, i.e., trapezoids. If $\boldsymbol{r}$ is general, then there is only one trapezoid, which must be planar and thus lies on the boundary of Figure 9.2 .

Proposition 9.5. For $t \in(0,1)$, the discriminant locus of the completely integrable system $\Psi_{t}$ inside $\operatorname{Int} B_{t}$ is

$$
\begin{aligned}
& \left\{\begin{array}{l|l}
\left(u_{1}, u_{2}, u_{3}, u_{4}\right) & \begin{array}{l}
\boldsymbol{r}=\left(\frac{u_{1}}{2}, \frac{u_{2}}{2}, \frac{u_{3}}{2}, \lambda-\frac{1}{2}\left(u_{1}+u_{2}+u_{3}\right)\right) \in H_{2} \\
u_{4}=(1-t)\left(r_{1}-r_{2}\right)^{2}-t\left(r_{1}-r_{4}\right)^{2}
\end{array}
\end{array}\right\} \\
& =\left\{\left(2 r_{1}, 2 r_{2}, 2 r_{3},(1-t)\left(r_{1}-r_{2}\right)^{2}-t\left(r_{1}-r_{4}\right)^{2}\right) \mid \boldsymbol{r}=\left(r_{i}\right) \in H_{2}\right\} .
\end{aligned}
$$

Proof. Let

$$
L_{t}(\boldsymbol{u})=\left\{x \mid\left(\psi_{12}(x), \psi_{23}(x), \psi_{34}(x), \psi_{t}(x)\right)=\left(u_{1}, u_{2}, u_{3}, u_{4}\right)\right\}
$$

be a Lagrangian fiber of $\Psi_{t}$ over an interior point $\boldsymbol{u}=\left(u_{1}, u_{2}, u_{3}, u_{4}\right) \in$ Int $B_{t}$. Since the moment map of the $\mathbb{T}_{U(4)}$-action on $\mathcal{O}_{\lambda}$ is given by

$$
\mu_{\mathbb{T}_{U(4)}}=\left(\psi_{12}, \psi_{23}, \psi_{34}, 2 \lambda-\left(\psi_{12}+\psi_{23}+\psi_{34}\right)\right),
$$

the fiber $L_{t}(\boldsymbol{u})$ lies in the level set $\mu_{\mathbb{T}_{U(4)}}^{-1}(2 \boldsymbol{r})$ for

$$
\boldsymbol{r}=\left(u_{1} / 2, u_{2} / 2, u_{3} / 2, \lambda-\left(u_{1}+u_{2}+u_{3}\right) / 2\right),
$$

and it is mapped to a level set of $\varphi_{t}$ under the symplectic reduction

$$
\pi: \mu_{\mathbb{T}_{U(4)}}^{-1}(2 \boldsymbol{r}) \rightarrow \mathcal{M}_{\boldsymbol{r}}
$$

Recall that the stabilizer at $x \in \operatorname{Gr}(2,4)$ of the action of the maximal torus $\mathbb{T}_{S U(4)}=\mathbb{T}_{U(4)} \cap S U(4)$ of $S U(4)$ is nontrivial exactly when

$$
x \in\left\{p_{12}=p_{34}=0\right\} \cup\left\{p_{13}=p_{24}=0\right\} \cup\left\{p_{14}=p_{23}=0\right\} .
$$

In this case, the corresponding point $\boldsymbol{\xi}=\pi(x) \in \mathcal{M}_{\boldsymbol{r}}$, regarded as a "quadrilateral" in $\mathbb{R}^{3}$, is contained in a straight line. If $x$ lies in $\left\{p_{12}=p_{34}=0\right\}$ (resp. $\left\{p_{14}=p_{23}=0\right\}$ ), one has $\boldsymbol{r} \in H_{1}$ (resp. $\boldsymbol{r} \in H_{3}$ ), and

$$
\begin{aligned}
\varphi \circ \pi(x) & =\left(\left(r_{1}+r_{2}\right)^{2},\left(r_{1}-r_{4}\right)^{2}\right)=\left(\max _{\mathcal{M}_{r}}\left(\varphi_{13}\right)^{2}, \min _{\mathcal{M}_{r}}\left(\varphi_{24}\right)^{2}\right) \\
(\text { resp. } \varphi \circ \pi(x) & \left.=\left(\left(r_{1}-r_{2}\right)^{2},\left(r_{1}+r_{4}\right)^{2}\right)=\left(\min _{\mathcal{M}_{r}}\left(\varphi_{13}\right)^{2}, \max _{\mathcal{M}_{r}}\left(\varphi_{24}\right)^{2}\right)\right)
\end{aligned}
$$

is the "lower-right" (resp. "upper-left") node of $\partial\left(\varphi\left(\mathcal{M}_{\boldsymbol{r}}\right)\right)$. Hence the fiber $L_{t}(\boldsymbol{u})$ over $\boldsymbol{u}=\left(u_{1}, u_{2}, u_{3}, u_{4}\right)=\left(2 r_{1}, 2 r_{2}, 2 r_{3}, c\right)$ for $c \in \operatorname{Int} I_{t, \boldsymbol{r}}$ is singular 
if and only if the line $\left\{\left(v_{1}, v_{2}\right) \mid(1-t) v_{1}-t v_{2}=c\right\}$ passes through the "lower-left" node

$$
\left(\left(r_{1}-r_{2}\right)^{2},\left(r_{1}-r_{4}\right)^{2}\right)=\left(\min _{\mathcal{M}_{r}}\left(\varphi_{13}\right)^{2}, \min _{\mathcal{M}_{r}}\left(\varphi_{24}\right)^{2}\right)
$$

of $\partial\left(\varphi\left(\mathcal{M}_{\boldsymbol{r}}\right)\right)$ as in Figure 9.8, which means that $\boldsymbol{r} \in H_{2}$ and

$$
c=(1-t)\left(r_{1}-r_{2}\right)^{2}-t\left(r_{1}-r_{4}\right)^{2} .
$$

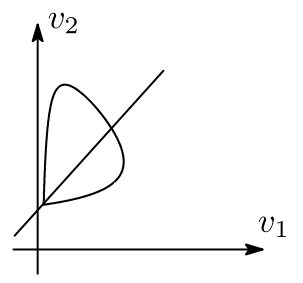

Figure 9.8: The image in $\partial\left(\varphi\left(\mathcal{M}_{\boldsymbol{r}}\right)\right)$ of a singular fiber $L_{t}(\boldsymbol{u})$.

Fix $t \in(0,1)$ and let $\boldsymbol{u}=\left(u_{1}, u_{2}, u_{3}, u_{4}\right)$ be a point in Int $B_{t}$ which is not on the discriminant of $\Psi_{t}$, so that $L_{t}(\boldsymbol{u}):=\Psi_{t}^{-1}(\boldsymbol{u})$ is a Lagrangian torus fiber. First we assume

$$
\varphi_{13}(\boldsymbol{u}) / \varphi_{24}(\boldsymbol{u}) \gg 1,
$$

so that $\pi\left(L_{t}(\boldsymbol{u})\right)$ is a small simple closed curve enclosing the point $\zeta_{\max } \in$ $\mathcal{M}_{\boldsymbol{r}} \cong \mathbb{P}^{1}$ at which $\max \varphi_{t}$ is attained. One can deform $\pi\left(L_{t}(\boldsymbol{u})\right)$ into a level set of $\varphi_{13}$ without crossing $x_{3}=0,-1$ by a Hamiltonian flow which sends $\zeta_{\max }$ to $x_{3}=\infty$. We may assume that the Hamiltonian is supported in a small neighborhood of the domain bounded by $\pi\left(L_{t}(\boldsymbol{u})\right)$. Note that if $r$ lies in the wall $H_{1}$, then one has $\zeta_{\max }=[0: 1: 1]$, which corresponds to $x_{3}=\infty$. Hence we may assume in this case that the support of the Hamiltonian does not contain the point $x_{3}=\infty$. We pull-back the Hamiltonian, and multiply a cut-off function supported near the level set $\mu_{\mathbb{T}_{U(4)}}^{-1}(\boldsymbol{r})$. Since it Poisson-commutes with the Hamiltonians of the $\mathbb{T}_{U(4)^{-}}$ action near $\mu_{\mathbb{T}_{U(4)}^{-1}}^{-1}(\boldsymbol{r})$, the induced Hamiltonian flow on $\operatorname{Gr}(2,4)$ sends $L_{t}(\boldsymbol{u})$ to a Lagrangian torus fiber $L_{\Gamma}\left(\boldsymbol{u}^{\prime}\right)$ of the integrable system $\Psi_{\Gamma}$ over some point $\boldsymbol{u}^{\prime}=\left(u_{12}, u_{23}, u_{34}, u_{13}\right) \in \operatorname{Int} \Delta_{\Gamma}$. We simultaneously deform the locus $\bigcup_{r} \pi^{-1}\left(\left\{\varphi_{t}=\max _{\mathcal{M}_{r}} \varphi_{t}\right\}\right)$ to

$$
\left\{\varphi_{13}=\max \varphi_{13}\right\}=\left\{p_{12} p_{34}=0\right\} \subset D,
$$

so the Lagrangian torus fiber does not cross this locus during the Hamiltonian isotopy from $L_{t}(\boldsymbol{u})$ to $L_{\Gamma}\left(\boldsymbol{u}^{\prime}\right)$. Note that $\Psi_{t}$ does not have a global action-angle coordinate because of the existence of the discriminant in Int $B_{t}$, and $\boldsymbol{u}^{\prime}$ is a local action-angle coordinate in the region $(9.45)$. 
One can further deform $\pi\left(L_{\Gamma}\left(\boldsymbol{u}^{\prime}\right)\right)$ to a circle $\left\{x_{3} \in \mathbb{C}|| x_{3} \mid=R\right\}$ by a Hamiltonian flow without crossing $x_{3}=0,-1, \infty$. The pull-back of the Hamiltonian gives a Hamiltonian flow $\operatorname{Gr}(2,4)$ which sends $L_{\Gamma}\left(\boldsymbol{u}^{\prime}\right)$ to a Lagrangian torus of the form $T_{r, R} \times T^{\prime}$ for a Clifford type Lagrangian torus $T_{r, R} \subset Y$ defined in 8.5 and a two-torus $T^{\prime} \subset\left(\mathbb{C}^{\times}\right)^{2}$.

Note that $L_{\Gamma}\left(\boldsymbol{u}^{\prime}\right)$ (and hence $T_{r, R} \times T^{\prime}$ ) can be deformed into a toric fiber in the toric variety $X_{\Gamma}$. The anti-canonical divisor $D$ can be deformed into the toric divisor in $X_{\Gamma}$, and thus the Maslov index of a holomorphic disk in $\left(\operatorname{Gr}(2,4), L_{\Gamma}\left(\boldsymbol{u}^{\prime}\right)\right)$ (and hence that in $\left.\left(\operatorname{Gr}(2,4), T_{r, R} \times T^{\prime}\right)\right)$ is twice the intersection number with $D$ (if the Lagrangian torus does not intersect $D)$. Then, by considering the projection $Y \times\left(\mathbb{C}^{\times}\right)^{2} \rightarrow \mathbb{C}$, no Lagrangian torus bounds holomorphic disks of non-positive Maslov index through the Lagrangian isotopy from $L_{t}(\boldsymbol{u})$ to $T_{r, R} \times T^{\prime}$, where we consider a compatible almost complex structure which induces the standard complex structure on $\mathbb{C}$ under the symplectic reduction. We also note that each holomorphic disk in $\operatorname{Gr}(2,4) \backslash D^{\prime}$ of Maslov index two bounded by $L_{t}(\boldsymbol{u})$ intersects the divisor $\left\{p_{14}=0\right\} \subset D$ at one point, and hence it descends to a disk in $Y$ of the same Maslov index. For $\beta \in \pi_{2}\left(Y, T_{r, R}\right)$, let $\tilde{\beta} \in \pi_{2}\left(\operatorname{Gr}(2,4), L_{t}(\boldsymbol{u})\right)$ denote its lift induced from an inclusion $Y \cong Y \times\{\mathrm{pt}\} \subset Y \times\left(\mathbb{C}^{\times}\right)^{2}$.

Lemma 9.6. The boundaries $\partial \tilde{\beta}_{1}, \partial \tilde{\beta}_{2} \in \pi_{1}\left(L_{t}(\boldsymbol{u})\right)$ of the lifts of $\beta_{1}, \beta_{2} \in$ $\pi_{2}\left(Y, T_{r, R}\right)$ defined in Example 8.3 are represented by Hamiltonian $S^{1}$-orbits of $\psi_{13}-\psi_{23}$ and $\psi_{13}-\psi_{12}-\psi_{23}-\psi_{34}$, respectively. The symplectic areas of $\tilde{\beta}_{1}, \tilde{\beta}_{2}$ are given by

$$
\begin{aligned}
& \omega\left(\tilde{\beta}_{1}\right)=u_{12}-\left(u_{12}+u_{23}-u_{13}\right)=u_{13}-u_{23}, \\
& \omega\left(\tilde{\beta}_{2}\right)=u_{13}-\left(u_{12}+u_{23}+u_{34}\right)+\lambda .
\end{aligned}
$$

Proof. Theorem 4.2 allows us to consider holomorphic disks in the central fiber $X_{\Gamma}$ of the toric degeneration

$$
\begin{aligned}
& \mathfrak{X}_{\Gamma} \\
& \downarrow \\
& \mathbb{C}
\end{aligned}=\left\{(\boldsymbol{p}, t) \in \mathbb{P}\left(\bigwedge^{2} \mathbb{C}^{4}\right) \times \mathbb{C} \mid p_{13} p_{24}=t p_{12} p_{34}+p_{14} p_{23}\right\}
$$

associated with $\Gamma$, instead of those in $\operatorname{Gr}(2,4)$. We recall a construction of the family $\Psi_{\Gamma}^{t}=\left(\psi_{i j}^{t}\right)_{\epsilon(i, j) \in \operatorname{Prn} \Gamma}$ of completely integrable systems connecting $\Psi_{\Gamma}$ and the toric moment map. Extend the actions of $\mathbb{T}_{U(4)}$ and $G(1,3) \subset$ $U(4)$ on $\operatorname{Gr}(2,4)$ to those on $\mathbb{P}\left(\bigwedge^{2} \mathbb{C}^{4}\right)$ in an obvious way, and let

$$
\begin{aligned}
\tilde{\mu}_{\mathbb{T}_{U(4)}}(\boldsymbol{p}) & =\left(\tilde{\psi}_{12}(\boldsymbol{p}), \tilde{\psi}_{23}(\boldsymbol{p}), \tilde{\psi}_{34}(\boldsymbol{p}), \tilde{\psi}_{14}(\boldsymbol{p})\right) \\
& =\frac{\lambda}{2 \sum\left|p_{i j}\right|^{2}}\left(\sum_{j \neq 1}\left|p_{1 j}\right|^{2}, \sum_{j \neq 2}\left|p_{2 j}\right|^{2}, \sum_{j \neq 3}\left|p_{3 j}\right|^{2}, \sum_{j \neq 4}\left|p_{4 j}\right|^{2}\right), \\
\tilde{\mu}_{G(1,3)}(\boldsymbol{p}) & =\frac{\lambda}{2 \sum\left|p_{i j}\right|^{2}}\left(\begin{array}{cc}
\sum_{j \neq 1}\left|p_{1 j}\right|^{2} & p_{23} \overline{p_{13}}+p_{24} \overline{p_{14}} \\
p_{13} \overline{p_{23}}+p_{14} \overline{p_{24}} & \sum_{j \neq 2}\left|p_{2 j}\right|^{2}
\end{array}\right)
\end{aligned}
$$


denote the moment maps of these actions. Then we obtain an extension $\tilde{\psi}_{13}: \mathbb{P}\left(\bigwedge^{2} \mathbb{C}^{4}\right) \rightarrow \mathbb{R}$ of $\psi_{13}$, which associates to $\boldsymbol{p}$ the maximum eigenvalues of $\tilde{\mu}_{G(1,3)}(\boldsymbol{p})$. The family of integrable systems $\Psi_{\Gamma}^{t}$ is given by the restrictions $\psi_{i j}^{t}=\left.\tilde{\psi}_{i j}\right|_{X_{t}}, \epsilon(i, j) \in \operatorname{Prn} \Gamma$ to each fiber $X_{t}=f_{\Gamma}^{-1}(t)$. Note that Poisson commutativity of $\Psi_{\Gamma}^{t}$ follows from the fact that the actions of $\mathbb{T}_{U(4)}$ and $G(1,3) \subset U(4)$ on $\mathbb{P}\left(\bigwedge^{2} \mathbb{C}^{2}\right)$ preserve each fiber $X_{t}$.

Using the defining equation

$$
p_{13} p_{24}=p_{14} p_{23}
$$

of the central fiber $X_{\Gamma}$, we have

$$
\begin{aligned}
\psi_{13}^{0} & =\frac{\lambda}{2 \sum\left|p_{i j}\right|^{2}}\left(\left|p_{12}\right|^{2}+\left|p_{13}\right|^{2}+\left|p_{14}\right|^{2}+\left|p_{23}\right|^{2}+\left|p_{24}\right|^{2}\right) \\
& =\frac{\lambda}{2}\left(1-\frac{\left|p_{34}\right|^{2}}{\sum\left|p_{i j}\right|^{2}}\right)
\end{aligned}
$$

which implies that Hamiltonian $S^{1}$-action of $\psi_{13}^{0}$ is given by

$$
e^{\sqrt{-1} \theta} \cdot \boldsymbol{p}=\left[p_{12}: p_{13}: p_{14}: p_{23}: p_{24}: e^{-\sqrt{-1} \theta} p_{34}\right] .
$$

We consider a deformation family

$$
\mathfrak{Y}_{\Gamma}=\left\{\left(x_{1}, x_{2}, x_{3}, t\right) \in \mathbb{C}^{3} \times \mathbb{C} \mid x_{1} x_{2}=t+x_{3}\right\} \rightarrow \mathbb{C}_{t}
$$

of $Y$ induced from the toric degeneration $(9.49)$, whose central fiber is given by

$$
Y_{\Gamma}=\left\{\left(x_{1}, x_{2}, x_{3}\right) \in \mathbb{C}^{3} \mid x_{1} x_{2}=x_{3}\right\} .
$$

The complement $X_{\Gamma} \backslash D_{\Gamma}^{\prime}$ of the divisor

$$
D_{\Gamma}^{\prime}=\left\{p_{12}=0\right\} \cup\left\{p_{23}=0\right\} \cup\left\{p_{34}=0\right\}
$$

on $X_{\Gamma}$ is identified with $Y_{\Gamma} \times\left(\mathbb{C}^{\times}\right)^{2}$, on which the Hamiltonian $S^{1}$-action of $\psi_{13}^{0}$ is given by

$$
e^{\sqrt{-1} \theta}\left(x_{1}, x_{2}, x_{3}, s_{1}, s_{2}\right)=\left(x_{1}, e^{\sqrt{-1} \theta} x_{2}, e^{\sqrt{-1} \theta} x_{3}, s_{1}, e^{-\sqrt{-1} \theta} s_{2}\right) .
$$

Since the lifts $\tilde{\beta}_{1}, \tilde{\beta}_{2}$, regarded as relative homotopy classes in $Y_{\Gamma} \times\left(\mathbb{C}^{\times}\right)^{2}$, are represented by holomorphic disks of the form $\left(x_{2}, s_{1}, s_{2}\right)=$ const. and $\left(x_{1}, s_{1}, s_{2}\right)=$ const., respectively, it follows from 9.31) and 9.60 that $\partial \tilde{\beta}_{1}$, $\partial \tilde{\beta}_{2}$ are represented by the following Hamiltonian $S^{1}$-orbits

$$
\begin{aligned}
\left(e^{\sqrt{-1} \theta} x_{1}, x_{2}, e^{\sqrt{-1} \theta} x_{3}, s_{1}, s_{2}\right) & \longleftrightarrow \psi_{13}^{0}-\psi_{23}^{0}, \\
\left(x_{1}, e^{\sqrt{-1} \theta} x_{2}, e^{\sqrt{-1} \theta} x_{3}, s_{1}, s_{2}\right) \longleftrightarrow & \psi_{13}^{0}+\psi_{14}^{0} \\
& =\psi_{13}^{0}-\psi_{12}^{0}-\psi_{23}^{0}-\psi_{34}^{0}+\text { const. }
\end{aligned}
$$


respectively. Here we recall the formula CO06, Theorem 8.1] for symplectic area of holomorphic disks Maslov index 2 in a toric manifold. Suppose that a holomorphic disk $w:\left(D^{2}, \partial D^{2}\right) \rightarrow\left(X_{\Gamma}, L_{0}(\boldsymbol{u})\right)$ of Maslov index 2 intersects a toric divisor $D_{\boldsymbol{v}}$ corresponding to the facet

$$
\left\{\boldsymbol{u} \in \Delta_{\Gamma} \mid \ell(\boldsymbol{u})=\langle\boldsymbol{v}, \boldsymbol{u}\rangle-\tau=0\right\}
$$

of the moment polytope. Then

$$
\left[w\left(\partial D^{2}\right)\right]=\boldsymbol{v} \in H^{1}\left(L_{0}(\boldsymbol{u}) ; \mathbb{Z}\right) \cong \mathbb{Z}^{2},
$$

and the symplectic ares of $w$ is given by

$$
\int_{D^{2}} w^{*} \omega=\ell(\boldsymbol{u})
$$

Comparing 9.61) and 9.62 with the defining inequalities

$\lambda$

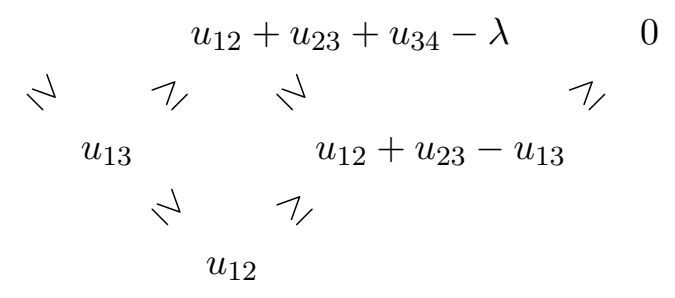

of the moment polytope $\Delta_{\Gamma}$, it follows that $\tilde{\beta}_{1}$ and $\tilde{\beta}_{2}$ intersect toric divisors corresponding to the facets of $\Delta_{\Gamma}$ defined by

$$
\begin{aligned}
& \ell_{1}(\boldsymbol{u})=u_{12}-\left(u_{12}+u_{23}-u_{13}\right)=u_{13}-u_{23}, \\
& \ell_{2}(\boldsymbol{u})=u_{13}-\left(u_{12}+u_{23}+u_{34}-\lambda\right),
\end{aligned}
$$

respectively. By topological reason, the results $\omega\left(\tilde{\beta}_{i}\right)=\ell_{i}(\boldsymbol{u})$ proved in $\left(X_{\Gamma}, L_{0}(\boldsymbol{u})\right)$ is true also in $\left(X_{t}, L_{t}(\boldsymbol{u})\right)$ for $t>0$.

Note that the defining functions (9.67) and (9.68) for $\Delta_{\Gamma}$ correspond to the following triangle inequalities

$$
\begin{aligned}
& u(2,3)-u(1,2) \leq u(1,3), \\
& u(3,4)-u(1,4) \leq u(1,3)
\end{aligned}
$$

in the coordinates defined in (3.33), respectively.

Next we assume that $\boldsymbol{u} \in \operatorname{Int} B_{t}$ satisfies $\varphi_{13}(\boldsymbol{u}) / \varphi_{24}(\boldsymbol{u}) \ll 1$. Then the image $\pi\left(L_{t}(\boldsymbol{u})\right) \subset \mathcal{M}_{\boldsymbol{r}}$ can be deformed into a level set of $\varphi_{24}$, which is a simple closed curve enclosing the point $\zeta_{3}=0$, and thus $L_{t}(\boldsymbol{u})$ is deformed into a fiber $\Psi_{\Gamma^{\prime}}\left(\boldsymbol{u}^{\prime \prime}\right)$ of the other completely integrable system $\Psi_{\Gamma}$ for some $\boldsymbol{u}^{\prime \prime}=\left(u_{12}, u_{23}, u_{34}, u_{24}\right)$. Since the point $\zeta_{3}=0$ corresponds to the origin in the $x_{3}$-plane, the fiber $L_{\Gamma^{\prime}}\left(\boldsymbol{u}^{\prime \prime}\right)$ can be deformed into $T_{r, R} \times T^{\prime}$ for a Chekanov type Lagrangian torus $T_{r, R} \subset Y$ and a two-torus $T^{\prime}$ in $\left(\mathbb{C}^{\times}\right)^{2}$. 
Lemma 9.7. The boundary $\partial \tilde{\beta}_{3} \in \pi_{1}\left(L_{t}(\boldsymbol{u})\right)$ of the lift of the class $\beta_{3} \in$ $\pi_{2}\left(Y, T_{r, R}\right)$ defined in Example 8.3 is represented by a Hamiltonian $S^{1}$-orbit of $\psi_{24}$, and the symplectic area of $\tilde{\beta}_{3}$ is given by

$$
\omega\left(\tilde{\beta}_{3}\right)=\lambda-u_{24} .
$$

Proof. We consider the central fiber $X_{\Gamma^{\prime}}$ of the toric degeneration

$$
\mathfrak{X}_{\Gamma^{\prime}}=\left\{(\boldsymbol{p}, t) \in \mathbb{P}\left(\bigwedge^{2} \mathbb{C}^{4}\right) \times \mathbb{C} \mid p_{13} p_{24}=p_{12} p_{34}+t p_{14} p_{23}\right\}
$$

associated with $\Gamma^{\prime}$, and let $\Psi_{\Gamma^{\prime}}^{0}=\left(\varphi_{i j}^{0}\right)_{\epsilon(i, j) \in \operatorname{Prn} \Gamma^{\prime}}$ denote the toric moment map on $X_{\Gamma^{\prime}}$ throughout this proof. Since $X_{\Gamma^{\prime}}$ is defined by

$$
p_{13} p_{24}=p_{12} p_{34},
$$

one has

$$
\begin{aligned}
\psi_{24}^{0} & =\frac{\lambda}{2 \sum\left|p_{i j}\right|^{2}}\left(\left|p_{12}\right|^{2}+\left|p_{13}\right|^{2}+\left|p_{23}\right|^{2}+\left|p_{24}\right|^{2}+\left|p_{34}\right|^{2}\right) \\
& =\frac{\lambda}{2}\left(1-\frac{\left|p_{14}\right|^{2}}{\sum\left|p_{i j}\right|^{2}}\right),
\end{aligned}
$$

which implies that its Hamiltonian $S^{1}$-action is

$$
e^{\sqrt{-1} \theta} \cdot \boldsymbol{p}=\left[p_{12}: p_{13}: e^{-\sqrt{-1} \theta} p_{14}: p_{23}: p_{24}: p_{34}\right] .
$$

Consider the family

$$
\mathfrak{Y}_{\Gamma^{\prime}}=\left\{\left(x_{1}, x_{2}, x_{3}, t\right) \in \mathbb{C}^{3} \times \mathbb{C} \mid x_{1} x_{2}=1+t x_{3}\right\}
$$

of affine varieties induced from (9.72), whose central fiber is given by

$$
Y_{\Gamma^{\prime}}=\left\{\left(x_{1}, x_{2}, x_{3}\right) \in \mathbb{C}^{3} \mid x_{1} x_{2}=1\right\} .
$$

For a divisor $D_{\Gamma^{\prime}}^{\prime}$ on $X_{\Gamma^{\prime}}$ defined by the same equation as $(9.59)$, the complement $X_{\Gamma^{\prime}} \backslash D_{\Gamma^{\prime}}^{\prime}$ is identified with $Y_{\Gamma^{\prime}} \times\left(\mathbb{C}^{\times}\right)^{2}$, on which the Hamiltonian $S^{1}$-action of $\psi_{24}^{0}$ is given by

$$
e^{\sqrt{-1} \theta}\left(x_{1}, x_{2}, x_{3}, s_{1}, s_{2}\right)=\left(x_{1}, x_{2}, e^{-\sqrt{-1} \theta} x_{3}, s_{1}, s_{2}\right) .
$$

From this and (9.31), the boundary $\partial \tilde{\beta}_{3}$, regarded as a class in the central fiber, is represented by a $S^{1}$-orbit of $\psi_{24}$. Since the moment polytope $\Delta_{\Gamma^{\prime}}$ is defined by

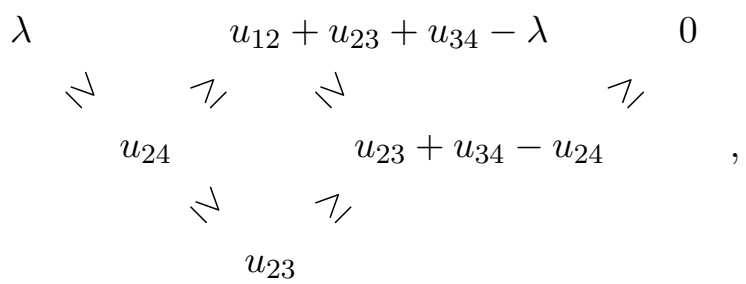


the holomorphic disk in $\tilde{\beta}_{3}$ intersects the toric divisor corresponding to the facet of $\Delta_{\Gamma^{\prime}}$ defined by

$$
\ell_{3}(\boldsymbol{u})=\lambda-u_{24},
$$

which corresponds to the triangle inequality

$$
u(2,4) \leq u(1,2)+u(1,4) .
$$

Lemma 9.7 follows the area formula 9.65 and invariance of symplectic areas under the deformation.

Finally we take a point $\boldsymbol{u} \in B_{t}$ on the wall, and consider the lift $\tilde{\alpha}$ of the class $\alpha \in \pi_{2}\left(Y, T_{r, R}\right)$ of Maslov index zero. Since $\alpha=\beta_{1}-\beta_{2}$, we have the following:

Lemma 9.8. The boundary $\partial \tilde{\alpha} \in \pi_{1}\left(L_{t}(\boldsymbol{u})\right)$ of the lift of $\alpha$ is represented by a Hamiltonian $S^{1}$-orbit of $\psi_{12}+\psi_{34}$, and the symplectic area of $\tilde{\alpha}$ is given by

$$
\omega(\tilde{\alpha})=u_{12}+u_{34}-\lambda .
$$

One can see this also from the fact that the class $\tilde{\alpha}$ is represented by a disk of the form $\left(x_{2}, s_{1}, s_{2}\right)=$ const., and thus (9.31) implies that the boundary of the disk is a Hamiltonian $S^{1}$-orbit of $\psi_{12}+\psi_{34}$.

From Lemmas 9.6, 9.7, 9.8, the functions $z_{\beta}(b)=T^{\omega(\beta)} \operatorname{hol}_{b}(\partial \beta)$ for $\beta=\tilde{\beta}_{1}, \tilde{\beta}_{2}, \tilde{\beta}_{3}, \tilde{\alpha}$ are given by

$$
\begin{aligned}
z_{\tilde{\beta}_{1}} & =\frac{y_{13}}{y_{23}}, \\
z_{\tilde{\beta}_{2}} & =\frac{q y_{13}}{y_{12} y_{23} y_{34}}, \\
z_{\tilde{\beta}_{3}} & =\frac{q}{y_{24}}, \\
z_{\tilde{\alpha}} & =\frac{y_{12} y_{34}}{q},
\end{aligned}
$$

where $q=T^{\lambda}$ for the Novikov parameter $T$, and therefore the coordinate change 9.3 gives

$$
z_{\tilde{\beta}_{3}}=z_{\tilde{\beta}_{1}}+z_{\tilde{\beta}_{2}}=z_{\tilde{\beta}_{2}}\left(1+z_{\tilde{\alpha}}\right),
$$

which coincides with the wall crossing formula 8.6 .

Remark 9.9. The Lagrangian torus fibers $L_{\Gamma}\left(\boldsymbol{u}_{0}\right)$ and $L_{\Gamma^{\prime}}\left(\boldsymbol{u}_{0}^{\prime}\right)$ above the centers

$$
\begin{aligned}
& \boldsymbol{u}_{0}=\left(u_{12}, u_{23}, u_{34}, u_{13}\right)=\left(\frac{\lambda}{2}, \frac{\lambda}{2}, \frac{\lambda}{2}, \frac{3 \lambda}{4}\right) \in \Delta_{\Gamma}, \\
& \boldsymbol{u}_{0}^{\prime}=\left(u_{12}, u_{23}, u_{34}, u_{24}\right)=\left(\frac{\lambda}{2}, \frac{\lambda}{2}, \frac{\lambda}{2}, \frac{3 \lambda}{4}\right) \in \Delta_{\Gamma^{\prime}}
\end{aligned}
$$


are monotone by [CK, Theorem B]. The fiber $L_{\Gamma}\left(\boldsymbol{u}_{0}\right)$ (resp. $\left.L_{\Gamma^{\prime}}\left(\boldsymbol{u}_{0}^{\prime}\right)\right)$ is contained in the complement $\operatorname{Gr}(2,4) \backslash D$ of the anti-canonical divisor $D$ given by $(9.26)$, and the image under the projection $\pi: \operatorname{Gr}(2,4) \backslash D \rightarrow \mathbb{C}_{x_{3}}$ is a simple closed curve enclosing $x_{3}=0,-1$ (resp. $\left.x_{3}=0\right)$. Note that $L_{\Gamma}\left(\boldsymbol{u}_{0}\right)$ and $L_{\Gamma^{\prime}}\left(\boldsymbol{u}_{0}^{\prime}\right)$ lie on the level set $\mu_{\mathbb{T}_{U(4)}}^{-1}(\lambda / 2, \ldots, \lambda / 2)$, and the intersection $\pi\left(L_{\Gamma}\left(\boldsymbol{u}_{0}\right)\right) \cap \pi\left(L_{\Gamma^{\prime}}\left(\boldsymbol{u}_{0}^{\prime}\right)\right)$, viewed as a subset in the polygon space, consists of two points corresponding to spatial quadrilaterals such that the configuration of vertices define a regular tetrahedron. The fiber $L=L_{\Gamma}(\lambda / 2, \ldots, \lambda / 2)$ of $\Psi_{\Gamma}$ on the boundary point

$$
\left(u_{12}, u_{23}, u_{34}, u_{13}\right)=(\lambda / 2, \lambda / 2, \lambda / 2, \lambda / 2) \in \partial \Delta_{\Gamma}
$$

is a Lagrangian $U(2) \cong S^{1} \times S^{3}$ (see [NU16, Proposition 2.7]). It is easy to see that $\varphi_{13}=0$ on $L$ and the image $\varphi(L)$ is a line segment connecting $x_{3}=0$ and $x_{3}=-1$ (see Figure 9.9p. Therefore the Lagrangian torus $L_{\Gamma^{\prime}}\left(\boldsymbol{u}_{0}^{\prime}\right)$ together with the inverse image in $L$ of the line segment connecting $x_{3}=$ -1 and the intersection point $\varphi\left(L_{\Gamma^{\prime}}\left(\boldsymbol{u}_{0}^{\prime}\right)\right) \cap \varphi(L)$ give a higher dimensional mutation configuration discussed in [PT17, Section 5]. Similarly, the fiber $L^{\prime}$ of $\Psi_{\Gamma^{\prime}}$ on the boundary point

$$
\left(u_{12}, u_{23}, u_{34}, u_{24}\right)=(\lambda / 2, \lambda / 2, \lambda / 2, \lambda / 2) \in \partial \Delta_{\Gamma^{\prime}}
$$

is a Lagrangian $U(2)$, whose image in the $x_{3}$-plane is $\varphi\left(L^{\prime}\right)=(-\infty,-1$ ]. Thus the pair of $L_{\Gamma}\left(\boldsymbol{u}_{0}\right)$ and the inverse image of the line segment connecting $x_{3}=-1$ and $\varphi\left(L_{\Gamma}\left(\boldsymbol{u}_{0}\right)\right) \cap \varphi\left(L^{\prime}\right)$ gives a mutation configuration. The

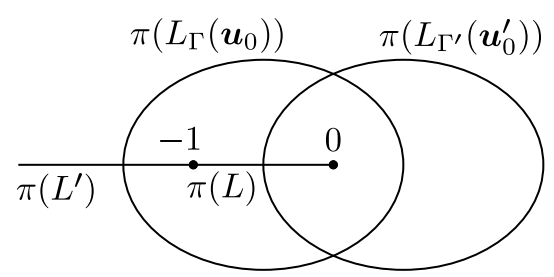

Figure 9.9: Images of Lagrangian fibers in the $x_{3}$-plane.

Lagrangian tori $L_{\Gamma}\left(\boldsymbol{u}_{0}\right)$ and $L_{\Gamma^{\prime}}\left(\boldsymbol{u}_{0}^{\prime}\right)$ are related by (the multiplication with a 2-torus of) a Lagrangian surgery studied in [Yau17, and the wall-crossing formula between them is obtained from [PT17, Theorem 5.7].

\section{Wall-crossing formula on general $\operatorname{Gr}(2, n)$}

In this section we complete the proof of Theorem 8.5.

We consider the family $f_{\Gamma^{\prime \prime}}: \mathfrak{X}_{\Gamma^{\prime \prime}} \rightarrow \mathbb{C}^{n-4}$ associated with the subdivision $\Gamma^{\prime \prime}$ given by common diagonals in $\Gamma$ and $\Gamma^{\prime}$, and let

$$
\Psi_{\Gamma}^{0}=\left(\left(\psi_{i j}^{0}\right)_{\epsilon(i, j) \in \operatorname{Prn} \Gamma^{\prime \prime}}, \psi_{a c}^{0}\right), \quad \Psi_{\Gamma^{\prime}}^{0}=\left(\left(\psi_{i j}^{0}\right)_{\epsilon(i, j) \in \operatorname{Prn} \Gamma^{\prime \prime}}, \psi_{b d}^{0}\right)
$$


be the completely integrable systems on the central fiber $X_{0}=f_{\Gamma^{\prime \prime}}^{-1}(\mathbf{0})$ obtained by deforming $\Psi_{\Gamma}$ and $\Psi_{\Gamma^{\prime}}$, respectively. Then $\Psi_{t}$ is deformed into the completely integrable system

$$
\Psi_{t}^{0}=\left(\left(\psi_{i j}^{0}\right)_{\epsilon(i, j) \in \operatorname{Prn} \Gamma^{\prime \prime}},(1-t)\left(\varphi_{a c}^{0}\right)^{2}-t\left(\varphi_{b d}^{0}\right)^{2}\right)
$$

on $X_{0}$, where we set $\varphi_{i j}^{0}=\psi_{i j}^{0}-\frac{1}{2} \sum_{k=i}^{j-1} \psi_{k, k+1}^{0}$, and thus Corollary 4.3 implies that we may work on $X_{0}$. For an open dense subset $Y^{\circ}$ of $Y$ defined by

$$
Y^{\circ}=\left\{\left(x_{1}, x_{2}, x_{3}\right) \in Y \mid x_{3} \neq 0\right\} \cong \mathbb{C}^{2} \backslash\left\{x_{1} x_{2}=1\right\},
$$

the isomorphisms given in Corollary 4.6 and $(9.28)$ yields

$$
X_{0} \backslash D_{0} \cong Y^{\circ} \times\left(\mathbb{C}^{\times}\right)^{2} \times \mathbb{T}_{\Gamma^{\prime \prime} \backslash \Gamma_{0}}^{\mathbb{C}} \cong Y^{\circ} \times\left(\mathbb{C}^{\times}\right)^{2 n-6}
$$

such that the restriction to $X_{0} \backslash D_{0}$ of the GIT quotient $X_{0}^{\mathrm{ss}} \rightarrow X_{0} / / \mathbb{T}_{\Gamma^{\prime \prime}}^{\mathbb{C}} \cong \mathbb{P}^{1}$ is identified with the projection

$$
f: Y^{\circ} \times\left(\mathbb{C}^{\times}\right)^{2 n-6} \rightarrow Y^{\circ} \rightarrow \mathbb{C}_{x_{3}}
$$

to the $x_{3}$-plane. Since $\mathbb{T}_{\Gamma^{\prime \prime}}^{\mathbb{C}}$ is the complexification of a torus $\mathbb{T}_{\Gamma^{\prime \prime}}$ generated by Hamiltonian flows of $\left(\psi_{i j}^{0}\right)_{\epsilon(i, j) \in \Gamma^{\prime \prime}}$, each Lagrangian torus fiber $L_{t}^{0}(\boldsymbol{u})$ of $\Psi_{t}^{0}$ is mapped by $f$ to a level set of $\varphi_{t}$ in a complex 1-dimensional polygon space $X_{0} / / \mathbb{T}_{\Gamma^{\prime \prime}}^{\mathbb{C}} \cong \operatorname{Gr}(2,4) / / \mathbb{T}_{U(4)}^{\mathbb{C}}$, which implies that $L_{t}^{0}(\boldsymbol{u})$ can be deformed into a Lagrangian torus of the form $T_{r, R} \times T^{\prime}$ for some $(r, R) \in \mathbb{R} \times \mathbb{R}_{>0}$ and a $(2 n-6)$-torus $T^{\prime}$ in $\left(\mathbb{C}^{\times}\right)^{2 n-6}$. We first assume that $T_{r, R}$ is of Clifford type. From the argument in the previous section, the lifts $\tilde{\beta}_{1}, \tilde{\beta}_{2} \in \pi_{2}\left(X_{0}, L_{t}^{0}(\boldsymbol{u})\right)$ of classes $\beta_{1}, \beta_{2}$ in $Y$ have symplectic areas

$$
\begin{aligned}
& \ell_{1}(\boldsymbol{u})=u_{a c}+u_{a b}-u_{b c}-\sum_{i=a}^{b-1} u_{i, i+1}, \\
& \ell_{2}(\boldsymbol{u})=u_{a c}+u_{a d}-u_{c d}-\sum_{i=a}^{c-1} u_{i, i+1},
\end{aligned}
$$

which correspond to the triangle inequalities

$$
\begin{gathered}
u(b, c)-u(a, b) \leq u(a, c), \\
u(c, d)-u(a, d) \leq u(a, c),
\end{gathered}
$$

respectively. On the other hand, in the case where $T_{r, R}$ is of Chekanov type, the symplectic area of $\tilde{\beta}_{3}$ is by

$$
\ell_{3}(\boldsymbol{u})=-u_{b d}+u_{a d}+u_{c d}-\sum_{i=a}^{b-1} u_{i, i+1}
$$


which corresponds to

$$
u(b, d) \leq u(c, d)+u(a, d) .
$$

Hence the functions $z_{\tilde{\beta}_{i}}$ are given by

$$
\begin{aligned}
& z_{\tilde{\beta}_{1}}=\frac{y_{a b} y_{a c}}{y_{b c} \prod_{i=a}^{b-1} y_{i, i+1}}, \\
& z_{\tilde{\beta}_{2}}=\frac{y_{a d} y_{a c}}{y_{c d} \prod_{i=a}^{c-1} y_{i, i+1}}, \\
& z_{\tilde{\beta}_{3}}=\frac{y_{a b} y_{a d}}{y_{b d} \prod_{i=a}^{b-1} y_{i, i+1}} .
\end{aligned}
$$

Since $z_{\tilde{\alpha}}$ corresponding to $\alpha=\beta_{1}-\beta_{2}$ is given by

$$
z_{\tilde{\alpha}}=\frac{z_{\tilde{\beta}_{1}}}{z_{\tilde{\beta}_{2}}}=\frac{y_{a b} y_{c d} \prod_{i=b}^{c-1} y_{i, i+1}}{y_{a d} y_{b c}},
$$

the coordinate change (5.17) is equivalent to the wall-crossing formula (9.88), which complete the proof.

\section{References}

[AAK16] Mohammed Abouzaid, Denis Auroux, and Ludmil Katzarkov, Lagrangian fibrations on blowups of toric varieties and mirror symmetry for hypersurfaces, Publ. Math. Inst. Hautes Études Sci. 123 (2016), 199-282. MR 3502098

[Aur07] Denis Auroux, Mirror symmetry and T-duality in the complement of an anticanonical divisor, J. Gökova Geom. Topol. GGT 1 (2007), 51-91. MR 2386535 (2009f:53141)

[Aur09]_ Special Lagrangian fibrations, wall-crossing, and mirror symmetry, Surveys in differential geometry. Vol. XIII. Geometry, analysis, and algebraic geometry: forty years of the Journal of Differential Geometry, Surv. Differ. Geom., vol. 13, Int. Press, Somerville, MA, 2009, pp. 1-47. MR MR2537081

[BCFKvS98] Victor V. Batyrev, Ionut Ciocan-Fontanine, Bumsig Kim, and Duco van Straten, Conifold transitions and mirror symmetry for Calabi-Yau complete intersections in Grassmannians, Nuclear Phys. B 514 (1998), no. 3, 640-666. MR MR1619529 (99m:14074)

[BCFKvS00] _ Mirror symmetry and toric degenerations of partial flag manifolds, Acta Math. 184 (2000), no. 1, 1-39. MR MR1756568 (2001f:14077) 
[BZ01] Arkady Berenstein and Andrei Zelevinsky, Tensor product multiplicities, canonical bases and totally positive varieties, Invent. Math. 143 (2001), no. 1, 77-128. MR MR1802793 (2002c:17005)

[CK] Yunhyung Cho and Yoosik Kim, Monotone Lagrangians in flag varieties, arXiv:1801.07554.

[CKO] Yunhyung Cho, Yoosik Kim, and Yong-Geun Oh, Lagrangian fibers of Gelfand-Cetlin systems, arXiv:1704.07213.

[CO06] Cheol-Hyun Cho and Yong-Geun Oh, Floer cohomology and disc instantons of Lagrangian torus fibers in Fano toric manifolds, Asian J. Math. 10 (2006), no. 4, 773-814. MR MR2282365 (2007k:53150)

[CPU16] Kwokwai Chan, Daniel Pomerleano, and Kazushi Ueda, Lagrangian torus fibrations and homological mirror symmetry for the conifold, Comm. Math. Phys. 341 (2016), no. 1, 135-178. MR 3439224

[EHX97] Tohru Eguchi, Kentaro Hori, and Chuan-Sheng Xiong, Gravitational quantum cohomology, Internat. J. Modern Phys. A 12 (1997), no. 9, 1743-1782. MR MR1439892 (99a:32027)

[FG06] Vladimir Fock and Alexander Goncharov, Moduli spaces of local systems and higher Teichmüller theory, Publ. Math. Inst. Hautes Études Sci. (2006), no. 103, 1-211. MR 2233852 (2009k:32011)

[FOOO09] Kenji Fukaya, Yong-Geun Oh, Hiroshi Ohta, and Kaoru Ono, Lagrangian intersection Floer theory: anomaly and obstruction, AMS/IP Studies in Advanced Mathematics, vol. 46, American Mathematical Society, Providence, RI, 2009. MR MR2553465

[FOOO10] L Lagrangian Floer theory on compact toric manifolds. I, Duke Math. J. 151 (2010), no. 1, 23-174. MR 2573826

[FST08] Sergey Fomin, Michael Shapiro, and Dylan Thurston, Cluster algebras and triangulated surfaces. I. Cluster complexes, Acta Math. 201 (2008), no. 1, 83-146. MR 2448067 (2010b:57032)

[FZ02] Sergey Fomin and Andrei Zelevinsky, Cluster algebras. I. Foundations, J. Amer. Math. Soc. 15 (2002), no. 2, 497-529 (electronic). MR 1887642 (2003f:16050) 
[FZ03] Cluster algebras. II. Finite type classification, Invent. Math. 154 (2003), no. 1, 63-121. MR 2004457 (2004m:17011)

[GM82] I. M. Gel'fand and R. D. MacPherson, Geometry in Grassmannians and a generalization of the dilogarithm, Adv. in Math. 44 (1982), no. 3, 279-312. MR 658730 (84b:57014)

[GS83] V. Guillemin and S. Sternberg, The Gel'fand-Cetlin system and quantization of the complex flag manifolds, J. Funct. Anal. 52 (1983), no. 1, 106-128. MR MR705993 (85e:58069)

[GSV05] Michael Gekhtman, Michael Shapiro, and Alek Vainshtein, Cluster algebras and Weil-Petersson forms, Duke Math. J. 127 (2005), no. 2, 291-311. MR 2130414 (2006d:53103)

[HK97] Jean-Claude Hausmann and Allen Knutson, Polygon spaces and Grassmannians, Enseign. Math. (2) 43 (1997), no. 1-2, 173-198. MR 1460127 (98e:58035)

[HK15] Megumi Harada and Kiumars Kaveh, Integrable systems, toric degenerations and Okounkov bodies, Invent. Math. 202 (2015), no. 3, 927-985. MR 3425384

[HMM11] Benjamin Howard, Christopher Manon, and John Millson, The toric geometry of triangulated polygons in Euclidean space, Canad. J. Math. 63 (2011), no. 4, 878-937. MR 2849001 (2012j:53110)

[Kly94] Alexander A. Klyachko, Spatial polygons and stable configurations of points in the projective line, Algebraic geometry and its applications (Yaroslavl', 1992), Aspects Math., E25, Vieweg, Braunschweig, 1994, pp. 67-84. MR 1282021 (95k:14015)

[KM96] Michael Kapovich and John J. Millson, The symplectic geometry of polygons in Euclidean space, J. Differential Geom. 44 (1996), no. 3, 479-513. MR 1431002 (98a:58027)

[MR] Robert Marsh and Konstanze Rietsch, The B-model connection and mirror symmetry for Grassmannians, arXiv:1307.1085.

[NNU10] Takeo Nishinou, Yuichi Nohara, and Kazushi Ueda, Toric degenerations of Gelfand-Cetlin systems and potential functions, Adv. Math. 224 (2010), no. 2, 648-706. MR 2609019

[NU14] Yuichi Nohara and Kazushi Ueda, Toric degenerations of integrable systems on Grassmannians and polygon spaces, Nagoya Math. J. 214 (2014), 125-168. MR 3211821 
[NU16] Floer cohomologies of non-torus fibers of the GelfandCetlin system, J. Symplectic Geom. 14 (2016), no. 4, 12511293. MR 3601889

[PT17] James Pascaleff and Dmitry Tonkonog, The wallcrossing formula and lagrangian mutations, arXiv preprint arXiv:1711.03209 (2017).

[Rie01] Konstanze Rietsch, Quantum cohomology rings of Grassmannians and total positivity, Duke Math. J. 110 (2001), no. 3, 523-553. MR 1869115 (2003c:14063)

[Rie08] , A mirror symmetric construction of $q H_{T}^{*}(G / P)_{(q)}$, Adv. Math. 217 (2008), no. 6, 2401-2442. MR 2397456 (2009f:14106)

[Rua01] Wei-Dong Ruan, Lagrangian torus fibration of quintic hypersurfaces. I. Fermat quintic case, Winter School on Mirror Symmetry, Vector Bundles and Lagrangian Submanifolds (Cambridge, MA, 1999), AMS/IP Stud. Adv. Math., vol. 23, Amer. Math. Soc., Providence, RI, 2001, pp. 297-332. MR MR1876075 (2002m:32041)

[RW] Konstanze Rietsch and Lauren Williams, Cluster duality and mirror symmetry for Grassmannians, arXiv:1507.07817.

[Sco06] Joshua S. Scott, Grassmannians and cluster algebras, Proc. London Math. Soc. (3) 92 (2006), no. 2, 345-380. MR 2205721 (2007e:14078)

[SS04] David Speyer and Bernd Sturmfels, The tropical Grassmannian, Adv. Geom. 4 (2004), no. 3, 389-411. MR 2071813 (2005d:14089)

[TW03] C. Teleman and C. Woodward, Parabolic bundles, products of conjugacy classes and Gromov-Witten invariants, Ann. Inst. Fourier (Grenoble) 53 (2003), no. 3, 713-748. MR 2008438 (2004g:14053)

[Wit95] Edward Witten, The Verlinde algebra and the cohomology of the Grassmannian, Geometry, topology, \& physics, Conf. Proc. Lecture Notes Geom. Topology, IV, Int. Press, Cambridge, MA, 1995, pp. 357-422. MR 1358625 (98c:58016)

[Yau17] Mei-Lin Yau, Surgery and isotopy of Lagrangian surfaces, Proceedings of the Sixth International Congress of Chinese Mathematicians. Vol. II, Adv. Lect. Math. (ALM), vol. 37, Int. Press, Somerville, MA, 2017, pp. 143-162. MR 3701933 
Yuichi Nohara

Department of Mathematics, School of Science and Technology,

Meiji University

1-1-1 Higashi-Mita, Tama-ku, Kawasaki-shi, Kanagawa 214-8571, Japan

e-mail address : nohara@meiji.ac.jp

Kazushi Ueda

Graduate School of Mathematical Sciences, The University of Tokyo,

3-8-1 Komaba, Meguro-ku, Tokyo, 153-8914, Japan

e-mail address : kazushi@ms.u-tokyo.ac.jp 